\title{
Intensity measures that reduce collapse capacity dispersion of P-delta vulnerable simple systems
}

\author{
Styliani Tsantaki ${ }^{1}$ Christoph Adam $^{1}$ (D) Luis F. Ibarra ${ }^{2}$
}

Received: 13 May 2015/ Accepted: 19 August 2016/Published online: 31 August 2016

(C) The Author(s) 2016. This article is published with open access at Springerlink.com

\begin{abstract}
The study evaluates two alternative seismic intensity measures (IMs) that reduce the collapse capacity dispersion of inelastic non-degrading single-degree-of-freedom (SDOF) systems vulnerable to the P-delta effect. This dispersion of collapse capacity is caused by record-to-record variability, which refers to frequency content variation of the ground motions used in the dynamic analyses. This reduction (of dispersion) is achieved utilizing efficient elastic pseudo-spectral acceleration based IMs. The first set of evaluated IMs is based on the spectral pseudo-acceleration averaged in a certain period interval between the structural period and an elongated period. The "optimal" lower bound of the period interval corresponds to the structural period of vibration, since naturally in an SDOF system no higher modes effects do exist. The "optimal" upper bound of the period interval for averaging, referred to as elongated period, is found to be 1.6 times the system period. The second IM considered in the study is the $5 \%$ damped spectral pseudo-acceleration at the system period in the presence of gravity loads, which is a single target IM. The most widely accepted IM, the $5 \%$ damped pseudo-spectral acceleration at the system period without P-delta, serves as the benchmark IM. The results show that both proposed IMs lead to a reduction of the collapse capacity dispersion compared to the benchmark IM outcomes. The IM based on the averaged spectral acceleration of the "optimal" period interval is more efficient up to a negative post-yield stiffness ratio of 0.45 , while the single target IM based on the system period in the presence of gravity loads is superior for extreme negative post-yield stiffness ratios larger than 0.45 . Additionally, the sufficiency and the scaling robustness property of the considered IMs with respect to the natural logarithm of the record-dependent individual collapse capacities is discussed for a wide range of structural configurations.
\end{abstract}

Christoph Adam

christoph.adam@uibk.ac.at

1 Unit of Applied Mechanics, University of Innsbruck, Technikerstr. 13, 6020 Innsbruck, Austria

2 Civil and Environmental Engineering, University of Utah, Salt Lake City, UT 84112, USA 
Keywords Efficient intensity measures · P-delta effect · Reduced record-to-record variability $\cdot$ Seismic collapse capacity

\section{Introduction}

The second generation of performance-based earthquake engineering (PBEE) methodologies (FEMA P-58-1 2012; FEMA P-695 2009) considers the inherent uncertainties in response prediction within a probabilistic framework. The variance on the seismic structural response depends on several factors such as the selected set of earthquake ground motions, the type of structure (e.g., mass and stiffness regularity), the considered response quantity, and the choice of the intensity measure (IM). In this paper, efficient IMs are evaluated to reduce the collapse capacity dispersion, due to record-to-record (RTR) variability, of highly inelastic non-deteriorating single-degree-of-freedom (SDOF) systems vulnerable to second-order effects.

Fundamental studies of the P-delta effect on inelastic SDOF systems subjected to earthquakes have been presented, for instance, by Husid (1967), Bernal (1987), and MacRae (1994). More recently, in the research group of the authors (Adam and Jäger 2012a; Jäger and Adam 2013) collapse capacity of P-delta sensitive SDOF systems has been assessed for a set of characteristic structural parameters. In Adam and Jäger (2012a) and Tsantaki et al. (2015) the presentation of the collapse capacity and its dispersion has been referred to as collapse capacity spectra, providing a compact and easily applicable tool for the practicing engineer.

The ground motion uncertainty is represented by one parameter or a vector of a few parameters related to a set of appropriately selected earthquake records, referred to as IM (Jalayer et al. 2012). The IM is the interface between seismology and earthquake engineering (Baker 2007). It quantifies the severity of a seismic event, and it serves as a scale factor for non-linear dynamic analysis. Since there is no unique definition of intensity of an earthquake record, several IMs have been proposed. They can be classified into (a) elastic ground motion based scalar IMs (Adam and Jäger 2012b), such as peak ground acceleration (PGA), peak ground velocity (PGV) and peak ground displacement (PGD); (b) elastic and inelastic spectral based IMs such as spectral acceleration and spectral displacement at the fundamental period of the structure, as well as spectral values related to higher modes effect or period elongation (Cordova et al. 2001; Haselton and Baker 2006; Luco and Cornell 2007; Bianchini et al. 2009; Kadas et al. 2011; Cantagallo et al. 2012; Vamvatsikos and Cornell 2005; Bojorquez and Iervolino 2011); and (c) vector valued IMs (e.g., Baker and Cornell 2005; Vamvatsikos and Cornell 2005). Currently, the most widely accepted IM is the $5 \%$ damped pseudo-spectral acceleration at the (fundamental) period of the structure, which serves in the present study as the benchmark IM.

Although many advanced IMs have been proposed, there are still a few limitations, such as the derivation of attenuation relations, the selection of the spectral values in case of higher modes and period elongation incorporation, their validation for several structural systems, etc. Based on information theory concepts, Jalayer et al. (2012) quantified the suitability of commonly used IMs against some others. The importance of the spectral shape consideration has been clearly demonstrated by Baker and Cornell (2006). Haselton (2009) evaluated several ground motion selection and modification methods utilizing a point of comparison methodology, and O'Donnell et al. (2013) investigated both 
experimental and analytical scaling methods for elastic systems. However, most of these studies did not address the collapse limit state, and were mainly focused on the efficiency of the considered IMs. The properties of sufficiency, scaling robustness and hazard computability have been studied by Shome and Cornell (1999), Luco and Cornell (2007), Bianchini et al. (2009), and Bojorquez and Iervolino (2011), among others.

In the present study, a slightly modified version of an IM proposed by Bianchini et al. (2009) is employed, based on the geometric mean of the pseudo-spectral acceleration over a certain period interval that yields the smallest dispersion of the collapse capacities. A preliminary study of Tsantaki and Adam (2013) showed that for the considered P-delta vulnerable SDOF systems this IM satisfies better the property of efficiency, compared with outcomes of benchmark studies (Adam and Jäger 2012a; Jäger and Adam 2013), where the $5 \%$ damped spectral pseudo-acceleration at the structural period has been used as IM. Eads et al. (2015) evaluated the efficiency and sufficiency of a similar IM for collapse prediction using almost 700 moment-resisting frame and shear wall structures. Also, Kazantzi and Vamvatsikos (2015) compared the effectiveness of several IMs based on the geometric mean concept superposing the spectral acceleration read at different logarithmically and linearly equally spaced periods. Lin et al. (2013a, b) demonstrated the importance of the conditioning period for the conditional spectrum in an intensity-based assessment, in contrast to a risk-based assessment.

The averaged period intervals depend on certain structural parameters, and system's level of inelasticity. Several codes and standards provide recommendations for the elongated period and higher mode effect intervals. For example, spectral matching period intervals of 0.2 to $2.0 T$ (Eurocode 82004 ), 0.4 to $1.5 T$ (NZSEE 2006) and 0.2 to $1.5 T$ (ASCE/SEI 41-13 2014) are proposed, with $T$ denoting the fundamental period of vibration. Recently, Katsanos et al. (2012) suggested to reduce the period interval specified in Eurocode 8 (i.e., $0.2 T<T<1.5 T$ ), at least for new buildings designed for low or moderate levels of ductility and low-to-medium stiffness degradation. Moreover, period elongation has been investigated numerically (Katsanos et al. 2012), as well as experimentally by testing full-scale models (Pinho and Elnashai 2000; Zembaty et al. 2006) and conducting response measurements of instrumented buildings (Mucciarelli et al. 2004; Clinton et al. 2006; Mucciarelli et al. 2012). However, most of the proposed code-bounds of the period intervals are based on expert elicitation, without referring to specific research studies. Thus, Sect. 3 presents a parametric study aimed to derive an "optimal" upper period bound that leads to the smallest collapse capacity dispersion of P-delta vulnerable SDOF systems. Subsequently, in Sect. 4 a single target IM is introduced for the collapse capacity assessment of P-delta vulnerable non-deteriorating SDOF systems. This IM is the $5 \%$ damped pseudo-acceleration at the period of vibration in the presence of gravity loads, $T_{S D O F}^{P \Delta}$ and referred to as "P-delta" IM, $S_{a}\left(T_{S D O F}^{P \Delta}\right)$. A comparison of the efficiency property of the utilized IMs is provided in Sect. 5, followed by a comparison of the sufficiency and scaling robustness property in Sect. 6.

\section{Definitions and framework}

\subsection{P-delta effect on an inelastic SDOF system}

In an inelastic SDOF system the gravity load generates a shear deformation of its hysteretic force-displacement relationship. Characteristic displacements (such as the yield displacement $x_{y}$ ) of this relationship remain unchanged, whereas the characteristic forces 
(such as the strength $f_{y}$ ) are reduced. As a result, the slope of elastic and post-elastic branch of deformation is decreased. The magnitude of this reduction can be expressed by means of the stability coefficient $\theta$ (MacRae 1994). The parameter $\theta$ is a function of the gravity load $P$, geometry, and stiffness. Based on an inverted mechanical pendulum of length $h$ and rotational base spring stiffness $k_{r}$ (Fig. 1a), the stability coefficient is (Adam and Jäger 2012a)

$$
\theta=\frac{P h}{k_{r}}, \quad P=\vartheta W
$$

where $W$ is the effective seismic weight, and $\vartheta=($ live load + dead load)/dead load. Figure 1 shows the P-delta effect on the non-dimensional hysteretic behavior of a SDOF system with non-deteriorating bilinear characteristics. In this example, the post-yield stiffness is negative, because the stability coefficient $\theta$ is larger than the hardening ratio $\alpha$.

A negative slope of the post-yield stiffness; i.e., $\theta-\alpha>0$, is a necessary condition for structural collapse under severe earthquakes. Adam and Jäger (2012a) showed that collapse of inelastic SDOF systems vulnerable to P-delta is mainly governed by the following parameters:

- the negative slope of the post-yield stiffness expressed by $\theta-\alpha$,

- the elastic structural period of vibration $T_{S D O F}$,

- the viscous damping coefficient $\zeta$ (usually $5 \%$ ), and

- the shape of the hysteretic loop.

In this paper SDOF systems with bilinear hysteretic behavior (Fig. 1b) are studied, considering a wide range of system configurations for various values of $T_{S D O F}, \theta-\alpha$, and $\zeta$.

\subsection{Collapse capacity variability}

Collapse capacity is defined as the maximum ground motion intensity at which the structure still maintains dynamic stability (Krawinkler et al. 2009). An Incremental Dynamic Analysis (IDA) is commonly used to predict collapse capacity, and consists of a

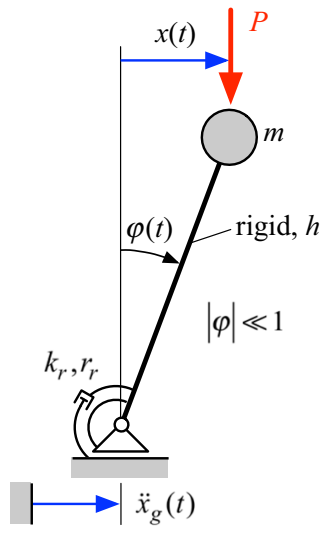

(a)

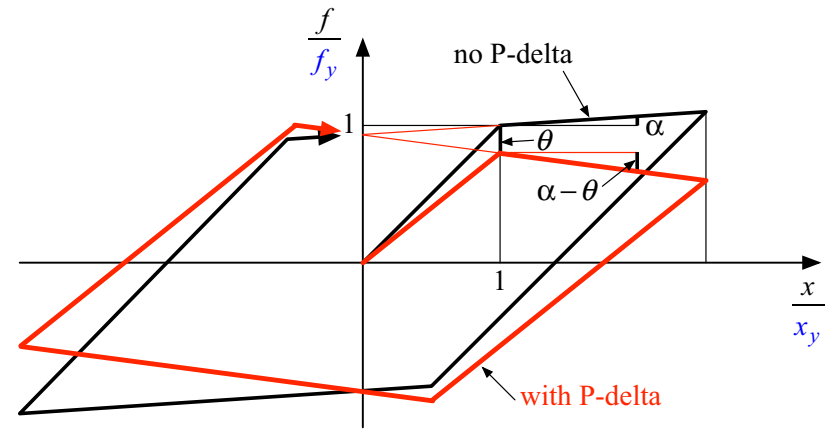

(b)

Fig. 1 a P-delta vulnerable SDOF system. b Normalized bilinear cyclic behavior with and without destabilizing effect of gravity loads (modified from Adam and Jäger 2012a) 
series of time history analyses in which the intensity of the ground motion record is monotonically increased (Vamvatsikos and Cornell 2002). In IDA curves, the IM is plotted against an engineering demand parameter (e.g., roof drift), until this parameter grows unbounded, indicating structural failure. The corresponding IM is referred to as collapse capacity of the considered structure.

When the IM is an acceleration quantity such as the PGA, or the $5 \%$ spectral pseudoacceleration at a target period of the structure, it is beneficial to relate the IM at collapse to the product of the base shear coefficient at onset of yield, $\gamma$, and acceleration gravity, $g$,

$$
\left.C C_{i}\right|_{I M}=\frac{\left.I M_{i}\right|_{\text {collapse }}}{g \gamma}, \gamma=\frac{f_{y}}{m g}
$$

In this equation, $f_{y}$ is the yield strength, and $m$ the mass of the SDOF system. $\left.C C_{i}\right|_{I M}$ is the relative collapse capacity of the considered SDOF system subjected to the $i$ th ground motion record.

Since RTR variability leads to different collapse capacities for different ground motion records, the collapse capacities are determined for all records of the considered ground motion set, and evaluated statistically. Shome and Cornell (1999) and Ibarra and Krawinkler $(2005,2011)$ provide good arguments for representing a set of collapse capacities by a log-normal distribution. The log-normal distribution of the individual collapse capacities, $C C_{j}$, can be characterized by the median, the 16 th, and 84 th percentiles of the collapse capacities denoted as $C C, C C_{P 16}$, and $C C_{P 84}$, respectively. In the following, the dispersion quantity $s^{*}$ (Adam and Jäger 2012a)

$$
s^{*}=\ln \sqrt{s_{u} s_{l}}, s_{u}=\frac{C C_{P 84}}{C C}, s_{l}=\frac{C C}{C C_{P 16}}
$$

is utilized as a representative measure of the variability of the individual collapse capacities. In the log-domain $s^{*}$ is related to the standard deviation: $\sigma \approx s^{*}$ (Limpert et al. 2001).

In this study, ground motion induced uncertainties are computed employing the 44 farfield ground motions of the FEMA P-695 far-field record set (FEMA P-695 2009), referred to as FEMA P-695-FF, and also known as PEER-NGA-FF and ATC63-FF. The records of the PEER- FEMA P-695-FF set originate from severe seismic events of magnitude between 6.5 and 7.6, and closest distance to the fault rupture larger than $10 \mathrm{~km}$. Only strike-slip and reverse sources are considered. The 44 records were recorded on NEHRP site classes C (soft rock) and D (stiff soil).

Exemplarily, Fig. 2 shows record dependent collapse capacity spectra based on the conventional IM $S_{a}\left(T_{S D O F}\right)$ for an SDOF system subjected to the FEMA P-695-FF record set. The SDOF system consists of a bilinear hysteretic loop, $\theta-\alpha=0.20$ and $\zeta=0.05$. Black lines with circle markers represent the corresponding 16th, 50th, and 84th percentile spectra.

\subsection{Evaluated intensity measures}

In Sects. 3, 4, and 5 of this paper the efficiency of the following three IMs is evaluated with respect to the collapse capacity dispersion attributed to RTR variability:

- the benchmark IM, $S_{a}\left(T_{S D O F}\right)$; i.e., the $5 \%$ damped pseudo-acceleration at the period of vibration $T_{S D O F}$,

- a set of "averaged" IMs, abbreviated by $S_{a, g m}$, where the $5 \%$ damped pseudoacceleration is averaged in a certain period interval, taking into account the period elongation due to the presence of gravity loads and inelastic deformations, and 


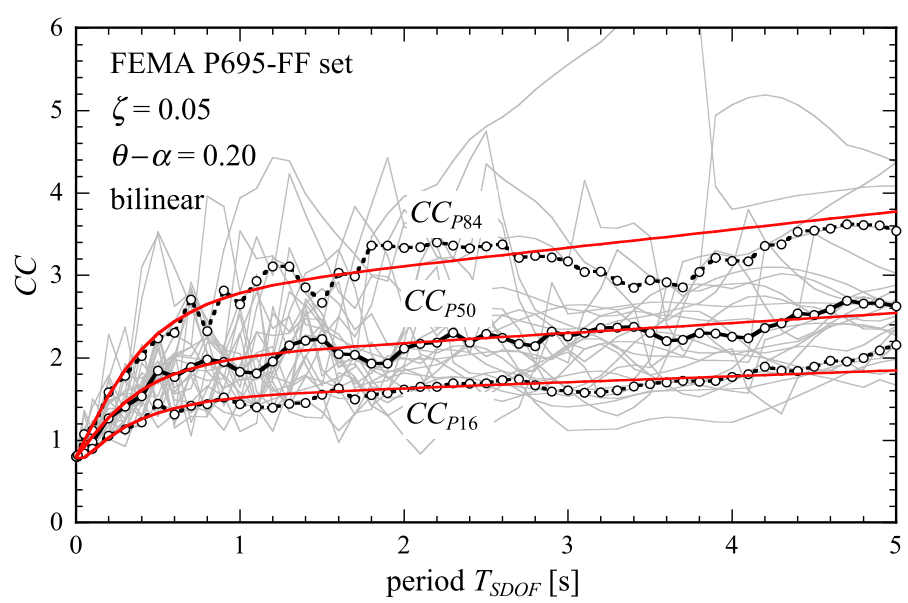

Fig. 2 FEMA P-695-FF 44 record dependent collapse capacity spectra (grey lines) based on the conventional IM $S_{a}\left(T_{S D O F}\right)$, corresponding statistical spectra (black lines with circle markers), and smooth empirical approximations (red lines)

- a single target IM, referred to as "P-delta" IM, $S_{a}\left(T_{S D O F}^{P \Delta}\right)$; i.e., the $5 \%$ damped pseudo-acceleration at the period of vibration in the presence of gravity loads, $T_{S D O F}^{P \Delta}$. This IM is introduced, because in the presence of gravity loads the initial period of vibration of an SDOF system, $T_{S D O F}$, is elongated by the factor $\sqrt{1 /(1-\theta)}: T_{S D O F}^{P \Delta}=$ $T_{\text {SDOF }} \sqrt{1 /(1-\theta)}$.

According to Bianchini et al. (2009) an appropriate IM should comply with three additional characteristics:

- Hazard computability; i.e., the IM quantifies appropriately the ground motion hazard at the site. The single target spectral pseudo-acceleration based IMs, $S_{a}\left(T_{S D O F}\right)$ and $S_{a}\left(T_{S D O F}^{P \Delta}\right)$, fulfill this property since attenuation relationships that describe the probability distribution of spectral pseudo-accelerations at single target periods are widely available. Seismic hazard analysis for the averaged IMs $S_{a, g m}$ can also be applied based on correlation equations between spectral accelerations at multiple periods derived by Baker and Jayaram (2008).

- Sufficiency; i.e., the IM is conditionally statistically independent of ground motion characteristics such as magnitude, distance, and epsilon, among others.

- Scaling robustness; i.e., the independence of IMs from scaling factors.

The sufficiency and the scaling robustness property of the utilized IMs is further discussed in Sect. 6.

\section{Averaged intensity measure}

\subsection{Definition}

In the present study, a slightly modified form of an averaged IM as defined in Bianchini et al. (2009) is implemented. This averaged IM is based on the geometric mean of the $5 \%$ damped spectral pseudo-accelerations $S_{a}$ over the period interval $\Delta T$, 


$$
\Delta T=T_{n}-T_{1}, \quad T_{n}>T_{1}
$$

between a lower bound period $T_{1}$ and an elongated upper bound period $T_{n}$ larger than $T_{1}$. In contrast to Bianchini et al. (2009), where $S_{a}$ is discretized at $10 \log$-spaced periods within $\Delta T$, in the present study $S_{a}$ is discretized at equally spaced periods $T_{i}$ within $\Delta T$,

$$
T_{i}=T_{1}+(i-1) \delta T, \quad i=1, \ldots, n, \delta T=\frac{\Delta T}{n-1}=\frac{T_{n}-T_{1}}{n-1}
$$

The discrete values of $S_{a}\left(T_{i}\right)(i=1, \ldots, n)$ are combined as proposed in Bianchini et al. (2009):

$$
S_{a, g m}\left(T_{1}, T_{n}\right)=\left(\prod_{i=1}^{n} S_{a}\left(T_{i}\right)\right)^{1 / n}
$$

$T_{i}$ is the $i$ th period in the set of $n$ periods $T_{1}, \ldots, T_{i}, \ldots, T_{n}$, and in general, it does not coincide with a system period.

\subsection{Sets of averaged intensity measures}

The effect of various spectral-averaged IMs $S_{a, g m}$ such as $S_{a, g m}\left(T_{1}=T_{S D O F}, T_{n}>T_{S D O F}\right)$, $S_{a, g m}\left(T_{1}<T_{S D O F}, T_{n}=T_{S D O F}\right)$ and $S_{a, g m}\left(T_{1}<T_{S D O F}, T_{n}>T_{S D O F}\right)$ on the collapse capacity dispersion $s^{*}$ is evaluated, and compared with the benchmark IM $S_{a}\left(T_{S D O F}\right)$. Note that all $S_{a, g m}$ based IMs are equivalent to the conventional IM $S_{a}\left(T_{S D O F}\right)$ for $\Delta T$ equal to 0 . For the analyses presented in this section, the SDOF systems have a bilinear cyclic behavior and viscous damping $\zeta$ of $5 \%$, unless otherwise indicated.

The first set of averaged IMs $S_{a, g m}\left(T_{1}=T_{S D O F}, T_{n}>T_{1}\right) \equiv S_{a, g m}\left(T_{S D O F}, T_{n}\right)$ has a lower bound period $T_{1}=T_{S D O F}$, and an upper bound period $T_{n}=T_{S D O F}+\Delta T$, which varies with the selected intervals $\Delta T$ of $0.4 \mathrm{~s}, 0.6 \mathrm{~s}, 0.8 \mathrm{~s}, 1.0 \mathrm{~s}, 1.2 \mathrm{~s}$, and $1.4 \mathrm{~s}$. The considered SDOF systems exhibit a negative post-yield stiffness ratio of $\theta-\alpha=0.20$, and system periods in the range $0<T_{S D O F} \leq 5.0 \mathrm{~s}$, equally spaced at increments of $0.1 \mathrm{~s}$. The collapse capacity is calculated at each discrete period $T_{S D O F}$ for all FEMA P-695-FF ground motions using an IDA approach, and the dispersion measure $s^{*}$ is evaluated using Eq. 3. This dispersion $s^{*}$ is plotted in Fig. 3 as a function of $T_{S D O F}$. The red solid line corresponds to $s^{*}$ for the original base case study using the conventional IM $S_{a}\left(T_{S D O F}\right)$, as presented in Adam and Jäger (2012a). Black lines represent outcomes utilizing the averaged IM $S_{a, g m}\left(T_{S D O F}, T_{n}\right)$ for various period intervals $\Delta T$. As observed, the conventional IM renders the smallest dispersion for very stiff systems, up to a period $T_{S D O F}=0.22 \mathrm{~s}$. As discussed in Jäger and Adam (2013), collapse capacity of a rigid system $\left(T_{S D O F}=0\right)$ does not exhibit aleatory uncertainty; i.e. $s^{*}=0$, when using the spectral acceleration at $T_{S D O F}$ as IM. However, the conventional IM leads to the largest dispersion for flexible systems with $T_{S D O F}>0.8 \mathrm{~s}$. For larger $T_{S D O F}$ the $S_{a, g m}\left(T_{S D O F}, T_{n}\right) \mathrm{IM}$ is more efficient, and leads to smaller $s^{*}$ values. For systems with periods $0.22 \mathrm{~s} \leq T_{S D O F} \leq 0.9 \mathrm{~s}$ the IM based on interval $\Delta T=0.4 \mathrm{~s}$ leads to the smallest dispersion. For more flexible systems with $T_{S D O F}>1.58 \mathrm{~s}$, the parameter $s^{*}$ is smallest for the IM with $\Delta T=1.4 \mathrm{~s}$., although the difference between the outcomes based on $\Delta T=1.2 \mathrm{~s}$ and $\Delta T=1.4 \mathrm{~s}$ is negligible. Note that for the considered SDOF systems the largest efficiency of the spectral averaged IM is for systems with periods $2.6 \mathrm{~s} \leq T_{S D O F} \leq 2.9 \mathrm{~s}$, where the reduction of $s^{*}$ is more than $55 \%$, compared to the outcome using the conventional IM. 


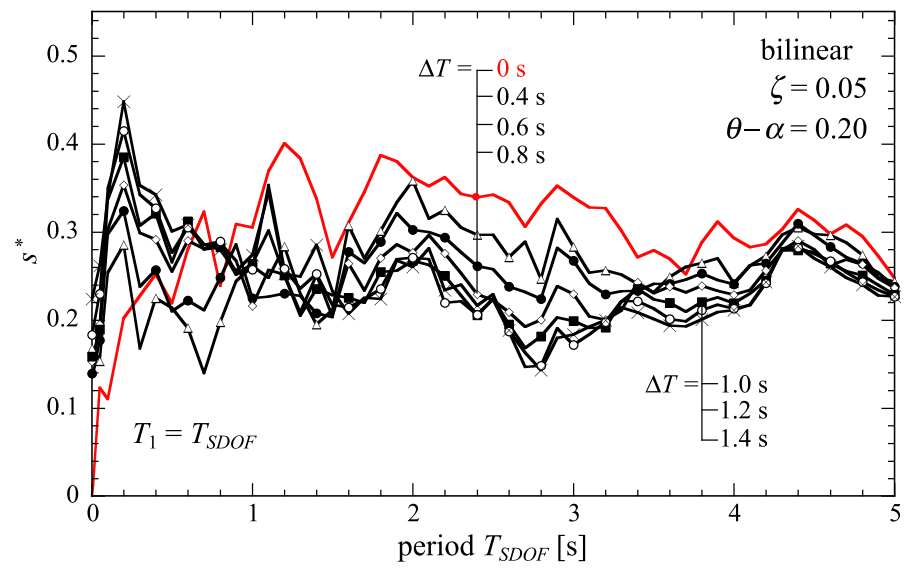

Fig. 3 Dispersion measure $s^{*}$ of the collapse capacity. IM: $S_{a, g m}\left(T_{S D O F}, T_{n}\right)$ with various intervals $\Delta T \geq 0$. Negative post-yield stiffness ratios $\theta-\alpha=0.20$

The study is extended considering a series of negative post-yield stiffness ratios $\theta-\alpha$ and additional intervals $\Delta T$ to find a general trend for the effect of the $S_{a, g m}\left(T_{S D O F}, T_{n}\right)$ averaged IM on $s^{*}$. The following values of $\theta-\alpha$ and $\Delta T$ values are assigned to the SDOF systems: $\theta-\alpha=0.04,0.06,0.08,0.10,0.20,0.30,0.40,0.50,0.60,0.80$; and $\Delta T=1.8 \mathrm{~s}$, $2.4 \mathrm{~s}, 3.0 \mathrm{~s}$, and $4.0 \mathrm{~s}$. The mean $\mathrm{E}_{\theta-\alpha}\left[s^{*}\right]$ of $s^{*}$ for each negative post-yield stiffness ratio $\theta-\alpha$ at a certain period $T_{S D O F}$ is determined as

$$
\mathrm{E}_{\theta-\alpha}\left[s^{*}\right]=\frac{1}{10} \sum_{k=1}^{10} s^{*}\left((\theta-\alpha)_{k}\right)
$$

In Fig. $4, \mathrm{E}_{\theta-\alpha}\left[s^{*}\right]$ is plotted against $T_{S D O F}$ for each IM (with different $\Delta T$ ) separately. The solid line in red corresponds to $\mathrm{E}_{\theta-\alpha}\left[s^{*}\right]$ based on the conventional IM $S_{a}\left(T_{S D O F}\right)$, whereas lines in black and blue represent the mean dispersion for different intervals $\Delta T$ of the IM $S_{a, g m}\left(T_{S D O F}, T_{n}\right)$. These results confirm the findings of Fig. 3. Except for very stiff structures, the conventional IM leads to a mean dispersion $\mathrm{E}_{\theta-\alpha}\left[s^{*}\right]$ of the collapse capacity of about 0.37 , while $\mathrm{E}_{\theta-\alpha}\left[s^{*}\right]$ based on $S_{a, g m}$ exhibits an average minimum of about 0.23 , when considering only the most efficient period intervals $\triangle T$ at different periods $T_{S D O F}$. Therefore, an averaged IM $S_{a, g m}\left(T_{S D O F}, T_{n}\right)$ can reduce the collapse capacity dispersion caused by RTR variability.

Additionally, for IMs $S_{a, g m}\left(T_{S D O F}, T_{n}\right)(0 \leq \Delta T \leq 1.4 \mathrm{~s})$ the mean of dispersion $s^{*}$, read at 53 discrete periods $T_{S D O F, j}(j=1,2, \ldots, 53)$ in the range $\left.0.001 \mathrm{~s} \leq T_{S D O F, j} \leq 5 \mathrm{~s}\right)$,

$$
\mathrm{E}_{T_{S D O F}}\left[s^{*}\right]=\frac{1}{53} \sum_{j=1}^{53} s^{*}\left(T_{S D O F, j}\right)
$$

is determined at each discrete negative post-yield stiffness ratio $\theta-\alpha$. Figure 5 displays $\mathrm{E}_{T_{S D O F}}\left[s^{*}\right]$ as a function of $\theta-\alpha$. In this representation, the conventional IM (i.e., $\Delta T=0$ ) leads to the poorest performance in the entire range of $\theta-\alpha$. For small $\theta-\alpha$ $(\theta-\alpha<0.10)$ the reduction of $\mathrm{E}_{T_{S D O F}}\left[s^{*}\right]$ based on $S_{a, g m}\left(T_{S D O F}, T_{n}\right)$ is small compared to that obtained for larger $\theta-\alpha(\theta-\alpha>0.10)$. These trends, as well as the increase of $\mathrm{E}_{T_{S D O F}}\left[s^{*}\right]$ as $\theta-\alpha$ becomes larger than 0.40 , will be discussed further in Sect. 6. 


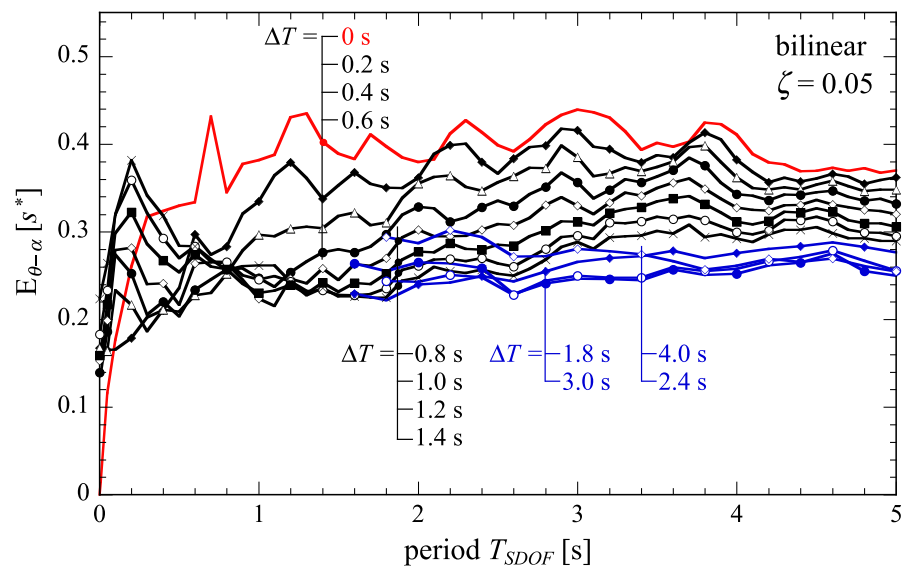

Fig. 4 Mean values of the dispersion measure $s^{*}$ with respect to the considered post-yield stiffness ratios $\theta-\alpha$. IM: $S_{a, g m}\left(T_{S D O F}, T_{n}\right)$ with various intervals $\Delta T \geq 0$

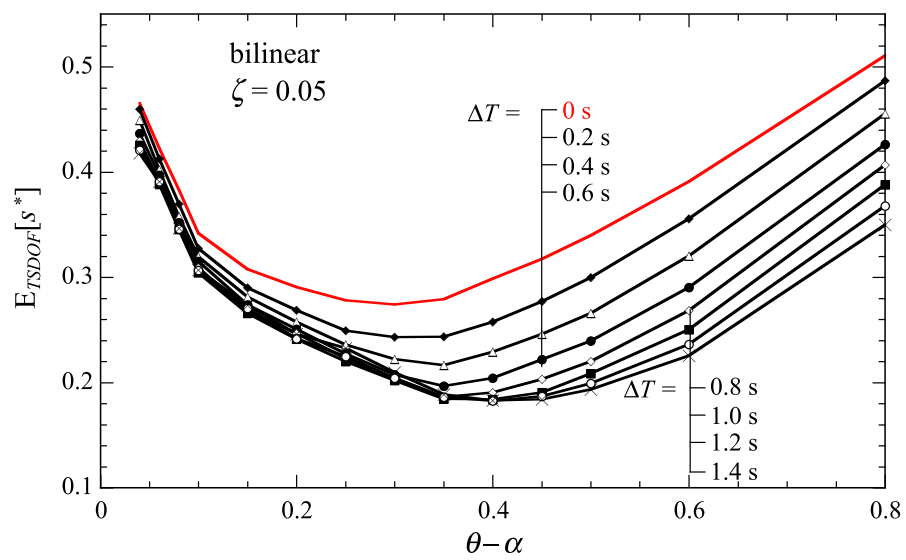

Fig. 5 Mean values of the dispersion measure $s^{*}$ with respect to the considered structural periods $T_{S D O F}$ as a function of the post-yield stiffness ratio $\theta-\alpha$. IM: $S_{a, g m}\left(T_{S D O F}, T_{n}\right)$ with various intervals $\Delta T \geq 0$

For SDOF systems the collapse capacity dispersion cannot be reduced by averaging the pseudo-acceleration for periods smaller than $T_{S D O F}$ because of the lack of higher mode effects. For the sake of completeness, however, $s^{*}$ is quantified assuming that the lower bound period $T_{1}$ of interval $\Delta T$ is smaller than the system period; i.e., $T_{1}<T_{S D O F}$, and the upper bound period $T_{n}=T_{S D O F}$. The corresponding set of IMs is $S_{a, g m}\left(T_{1}<T_{S D O F}, T_{n}=\right.$ $\left.T_{S D O F}\right)$ referred to as $S_{a, g m}\left(T_{1}, T_{S D O F}\right)$. As shown in Fig. 6, the use of averaged IM $S_{a, g m}$ in which $T_{1}<T_{S D O F}$ leads to a larger collapse capacity variability compared to that obtained from the conventional IM $S_{a}\left(T_{S D O F}\right)$.

As expected, the use of sets of IMs $S_{a, g m}\left(T_{1}<T_{S D O F}, T_{n}>T_{S D O F}\right) \equiv S_{a, g m}\left(T_{1}, T_{n}\right)$, such as $S_{a, g m}\left(0.2 T_{S D O F}, 1.6 T_{S D O F}\right)$, leads to larger collapse capacity dispersions compared to those based on IM $S_{a, g m}\left(T_{S D O F}, 1.6 T_{S D O F}\right)$, as shown in Fig. 7 . The use of IM $S_{a, g m}\left(T_{S D O F}, 1.6 T_{S D O F}\right)$ leads to the smallest $s^{*}$ for most periods. Also, the use of an IM 


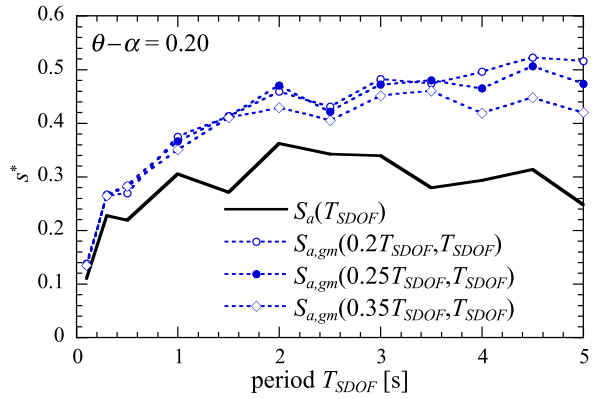

(a)

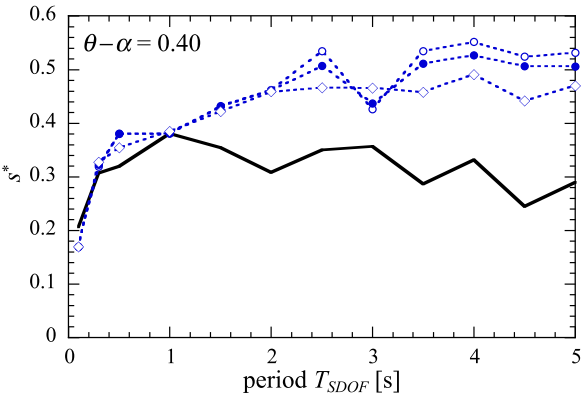

(b)

Fig. 6 Dispersion measure $s^{*}$ of the collapse capacity. IMs: $S_{a}\left(T_{S D O F}\right), S_{a, g m}\left(T_{1}, T_{S D O F}\right)$. Negative postyield stiffness ratio a $\theta-\alpha=0.20, \mathbf{b} \theta-\alpha=0.40$

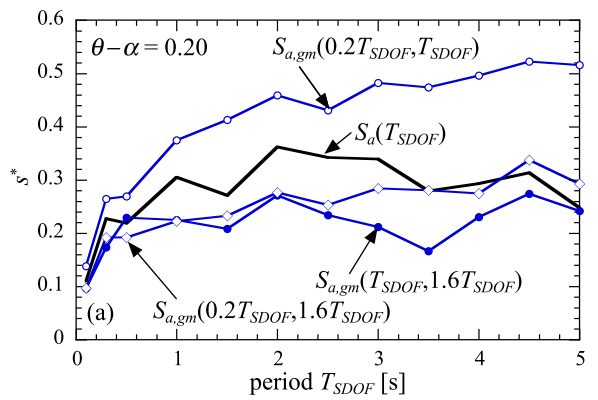

(a)

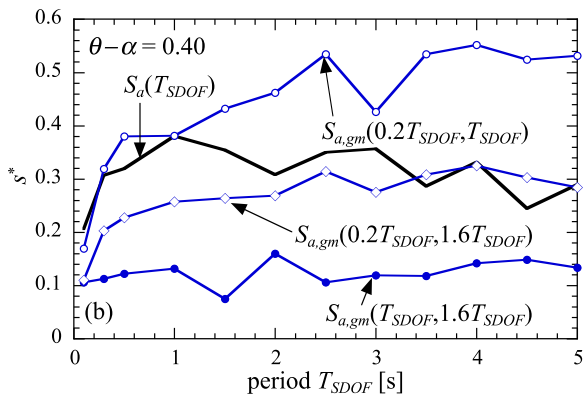

(b)

Fig. 7 Dispersion measure $s^{*}$ of the collapse capacity. Various intensity measures. Negative post-yield stiffness ratio a $\theta-\alpha=0.20, \mathbf{b} \theta-\alpha=0.40$

with the period interval $\Delta T$ below the system period $\left(T_{1}=0.2 T_{S D O F}\right.$ and $\left.T_{n}=T_{S D O F}\right)$ performs poor with respect to $s^{*}$, except for very stiff systems.

\section{3 "Optimal" averaged intensity measure}

Based on the above results, an extensive parametric IDA study has been carried out to identify the upper bound period limit $T_{n}=T_{S D O F}+\Delta T>T_{S D O F}$ that leads to the "optimal" (i.e., the minimum) dispersion $s^{*}$ of collapse capacities.

According to Fig. 4, there is no "optimal" IM with constant $\Delta T$ for the entire range of system periods, because the "optimal" interval $\Delta T$ is a function of $T_{S D O F}$. That is, the larger the elastic period $T_{S D O F}$ becomes, the larger the elongated inelastic period, and consequently, the larger is the required $\Delta T$ to render the minimum achievable dispersion measure $s^{*}$. To obtain a relation between $T_{S D O F}$ and the "optimal" interval $\Delta T_{o p t}$ $\left(\min s^{*} \rightarrow \Delta T_{\text {opt }}\right.$ ), the dispersion measure $s^{*}$ is determined as a function of $\Delta T$ for discrete system periods. Figure 8 shows the mean dispersion $\mathrm{E}_{\theta-\alpha}\left[s^{*}\right]$ for nine system periods $\left(T_{S D O F}=0.2,0.5,0.7,1.0,1.5,2.1,3.0,4.0\right.$, and $\left.5.0 \mathrm{~s}\right)$ plotted against interval $\Delta T$ of the averaged IM $S_{a, g m}\left(T_{1}=T_{S D O F}, T_{n}>T_{S D O F}\right)$ and the conventional IM $S_{a}\left(T_{S D O F}\right)$ (i.e., $\Delta T=0) . \mathrm{E}_{\theta-\alpha}\left[s^{*}\right]$ is determined comprising the complete set of considered post-yield stiffness ratios $\theta-\alpha$ (i.e., $0.04,0.06,0.08,0.10,0.20,0.30,0.40,0.50,0.60,0.80$ ). 


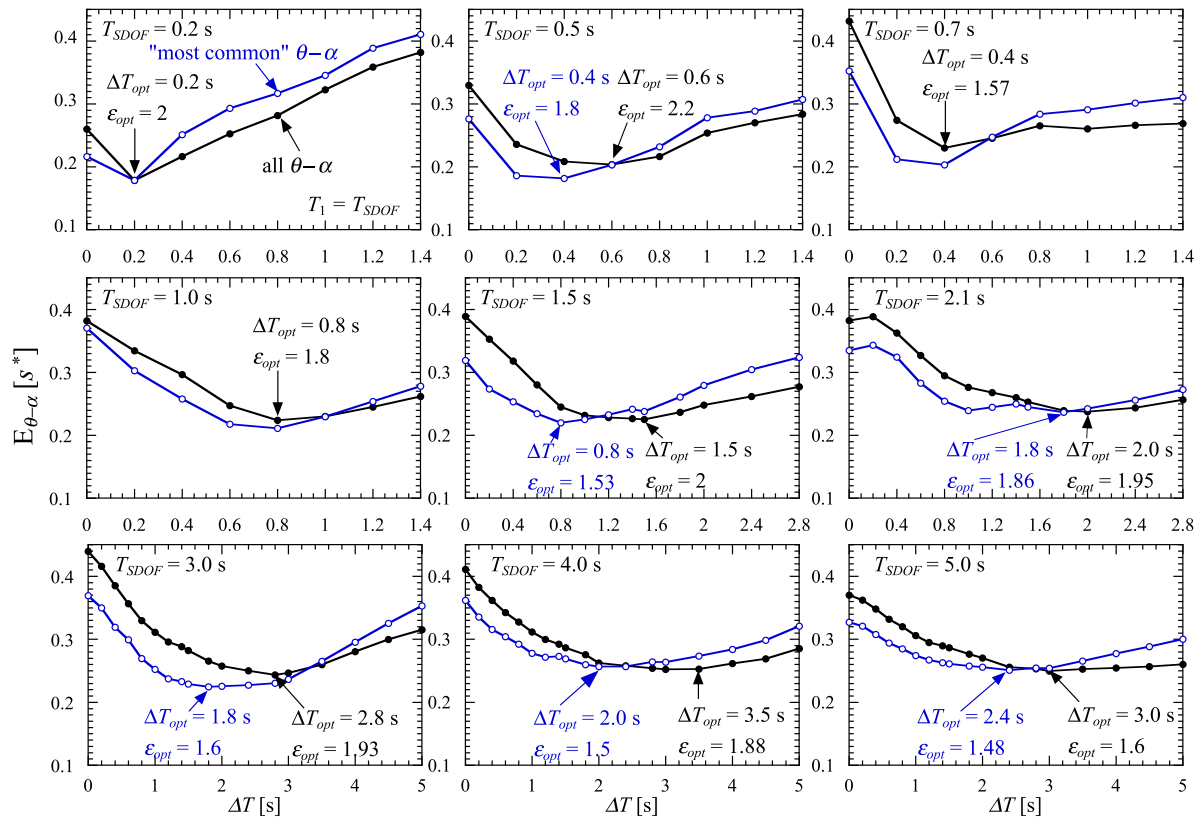

Fig. 8 Mean values of dispersion $s^{*}$ with respect to the negative post-yield stiffness ratios $\theta-\alpha$ as a function of period interval $\Delta T$ for nine discrete structural periods $T_{S D O F}$. Black curves: mean from all considered discrete values of $\theta-\alpha$. Blue curves: mean from discrete values of $\theta-\alpha$ in the range of $0.08 \leq \theta-\alpha \leq 0.40$. Intensity measure $S_{a, g m}\left(T_{S D O F}, T_{n}\right)$ with various intervals $\Delta T \geq 0$

Alternatively, only the collapse capacities for $\theta-\alpha$ in the range of $0.08 \leq \theta-\alpha \leq 0.40$ (referred to as "most common" $\theta-\alpha$ ) are used to calculate the mean dispersion measure $\mathrm{E}_{\theta-\alpha}\left[s^{*}\right]$. In the latter set of $\theta-\alpha$, extreme (unlikely) values, which might distort the general trend of the response behavior, are excluded. Exemplarily for a system with $T_{S D O F}=1.5 \mathrm{~s}$, Fig. 9 shows both the dispersion $s^{*}$ for each discrete $\theta-\alpha$ value and the corresponding $\mathrm{E}_{\theta-\alpha}\left[s^{*}\right]$ values. It can be observed that the trend of the dispersion for systems with an extremely large negative post-yield stiffness ratio of $\theta-\alpha=0.80$ is very different from the other depicted cases. The results of Fig. 8 confirm that the "optimal" interval $\Delta T_{\text {opt }}$ strongly depends on the system period $T_{S D O F}$, and that the optimal interval $\Delta T_{\text {opt }}$ becomes larger with increasing $T_{S D O F}$. In all the plots of Fig. 8, the interval $\Delta T_{o p t}$ that leads to the minimum mean dispersion is specified. For example, for $T_{S D O F}=1.0 \mathrm{~s}$ the minimum mean dispersion is 0.21 (all $\theta-\alpha$ ), compared to 0.38 for the benchmark IM (i.e., $\Delta T=0$ ). For the minimum mean $\mathrm{E}_{\theta-\alpha}\left[s^{*}\right]$ the corresponding $\Delta T_{o p t}$ is about $0.8 \mathrm{~s}$. The ratio $\varepsilon_{o p t}$ between the "optimal" upper bound period $T_{n, o p t}=T_{S D O F}+\Delta T_{\text {opt }}$ and period $T_{1}=T_{S D O F}$ is

$$
\varepsilon_{o p t}=\frac{T_{n, o p t}}{T_{S D O F}}
$$

This ratio $\varepsilon_{\text {opt }}$ lies between 1.4 and 2.0 for all the evaluated periods.

Figure 10 shows ratio $\varepsilon_{o p t}$ as a function of $T_{S D O F}$ for three stiffness ratios $\theta-\alpha$ of 0.10 , 0.20 and 0.40 , and for the mean of $s^{*}$ with respect to these $\theta-\alpha$ values. As observed, for rigid SDOF systems $\varepsilon_{\text {opt }}$ is 1 , because the dispersion is 0 when using the benchmark IM 


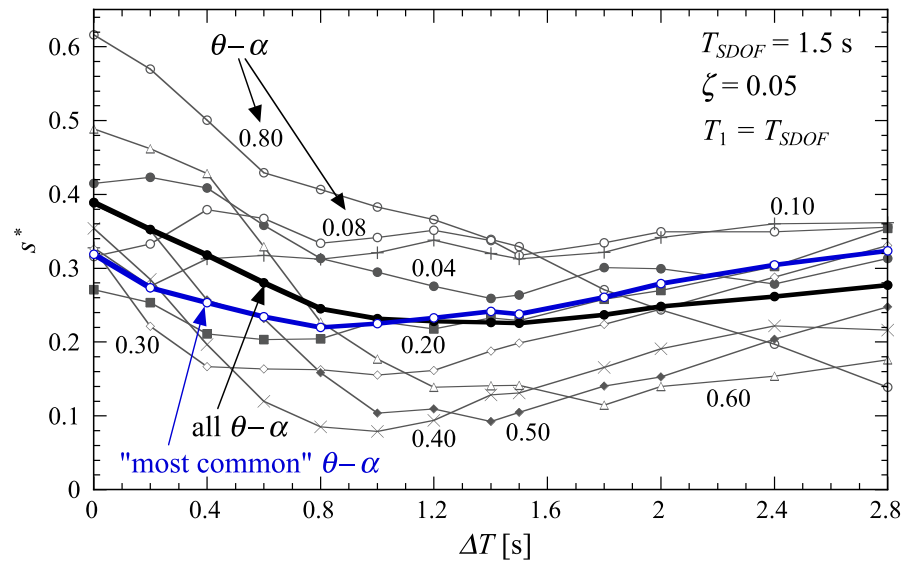

Fig. 9 Dispersion measure $s^{*}$ for various negative post-yield stiffness ratios $\theta-\alpha$ as a function of period interval $\Delta T$. Black graph: mean from all considered discrete values of $\theta-\alpha$. Blue graph: mean from discrete values of $\theta-\alpha$ in the range of $0.08 \leq \theta-\alpha \leq 0.40$. Intensity measure $S_{a, g m}\left(T_{S D O F}, T_{n}\right)$ with various intervals $\Delta T \geq 0$

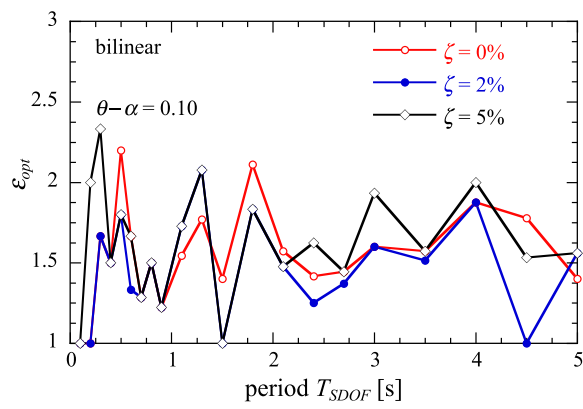

(a)

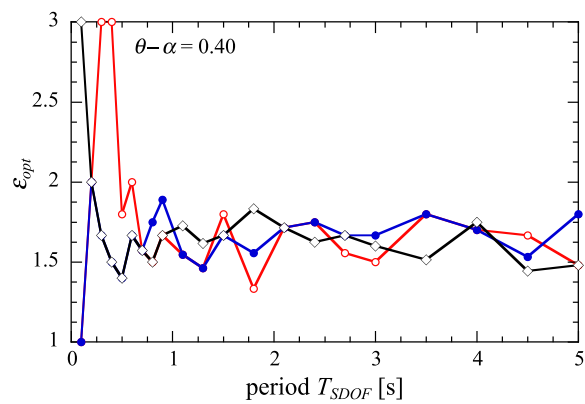

(c)

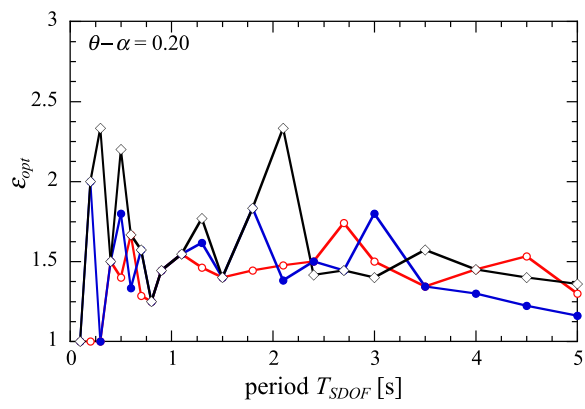

(b)

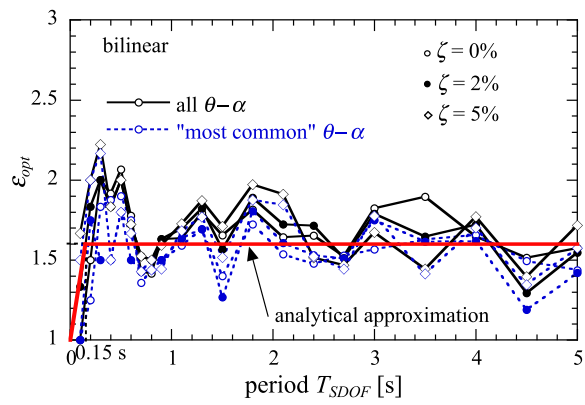

(d)

Fig. 10 Ratio $\varepsilon_{\text {opt }}$ of the "optimal" elongated period $T_{n, o p t}$ and the system period $T_{S D O F}$ leading to the minimum dispersion $s^{*}$. Viscous damping $\zeta=0,2$, and $5 \%$. a-c Ratios for individual negative post-yield stiffness ratios as specified, $\mathbf{d}$ ratios based on $\mathrm{E}_{\theta-\alpha}\left[s^{*}\right]$ 


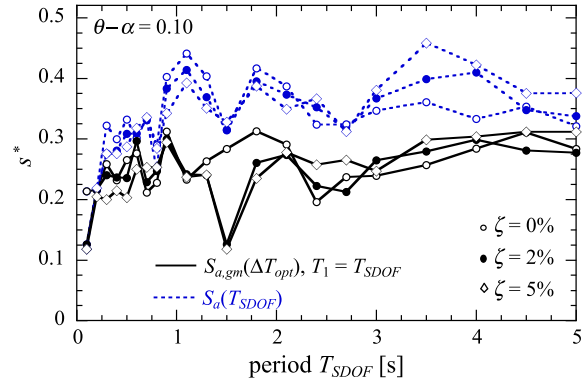

(a)

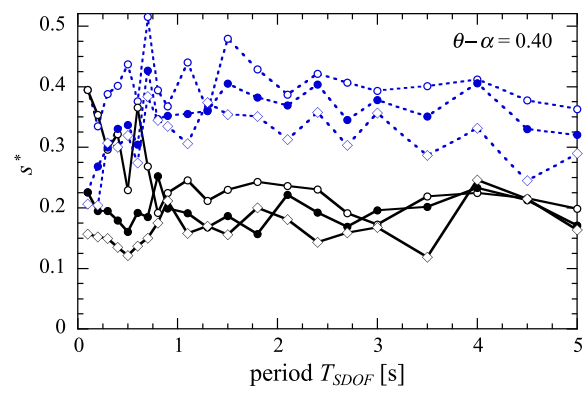

(c)

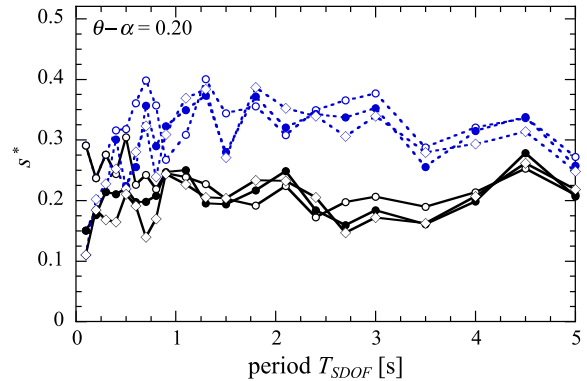

(b)

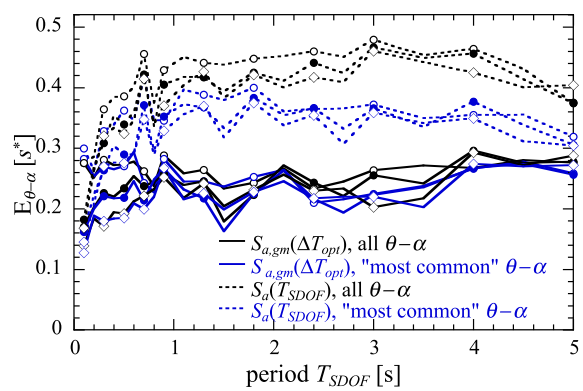

(d)

Fig. 11 Smallest dispersion measure $s^{*}$. Intensity measures: $S_{a, g m}\left(T_{S D O F}, T_{n, o p t}\right), S_{a}\left(T_{S D O F}\right)$. Viscous damping $\zeta=0,2$, and $5 \%$. a-c $s^{*}$ for individual negative post-yield stiffness ratios as specified; $\mathbf{d}$ mean $\mathrm{E}_{\theta-\alpha}\left[s^{*}\right]$

$S_{a}\left(T_{S D O F}\right)$ (Jäger and Adam 2013). Then follows a sharp rise of $\varepsilon_{o p t}$ for stiff systems with $T_{S D O F}<0.5 \mathrm{~s}$. In this period range $\varepsilon_{\text {opt }}$ can be larger than 2 . For periods $T_{S D O F}>0.8 \mathrm{~s}, \varepsilon_{\text {opt }}$ fluctuates around 1.6. For smaller values of $\theta-\alpha$ the fluctuation of $\varepsilon_{o p t}$ around 1.6 is larger. The mean of $s^{*}$ with respect to outcomes for different $\theta-\alpha$ values exhibits the same global behavior. Figure 10 reveals that the impact of $\theta-\alpha$ on $\varepsilon_{\text {opt }}$ is insignificant. In addition to the evaluation of $5 \%$ damped SDOF systems, Fig. 10d also presents results for undamped and $2 \%$ damped systems. As can be seen, the fluctuation around 1.6 is preserved.

Figure 11 compares the dispersion measure $s^{*}$ that corresponds to the optimal period interval $\Delta T_{o p t}$ (and thus to $T_{n, o p t}$ and to $\varepsilon_{\text {opt }}$ ) to the dispersion obtained from the benchmark $\operatorname{IM} S_{a}\left(T_{S D O F}\right)$ for the same systems. According to Fig. 11d the mean $\mathrm{E}_{\theta-\alpha}\left[s^{*}\right]$ is on average about 0.23 for the entire period interval $T_{S D O F}$. This value remains the same considering the complete set of $\theta-\alpha$ and, alternatively, the set including the "most common" $\theta-\alpha$. The mean dispersion of collapse capacities based on the benchmark IM $S_{a}\left(T_{S D O F}\right)$; i.e., $\mathrm{E}_{\theta-\alpha}\left[s^{*}\right]$, is on average about 0.37 , which is about $60 \%$ larger than the dispersion based on $\Delta T_{o p t}$, proving the superiority of the averaged IM $S_{a, g m}$ in reducing the effect of RTR variability on the collapse capacity dispersion. Furthermore, the results of Fig. 11 show that, not only the ratio $\varepsilon_{\text {opt }}$, but also the dispersion measure $s^{*}$ is globally unaffected from viscous damping for all utilized IMs. The outcomes for individual negative stiffness ratios $\theta-\alpha$ confirm these findings. It is also shown that dispersion measure $s^{*}$ is practically independent from $\theta-\alpha$. 
Based on the above observations, the following analytical approximation for $\varepsilon_{\text {opt }}$, denoted as $\varepsilon_{\alpha}$, is proposed:

$$
\varepsilon_{\alpha}\left(T_{S D O F}\right)= \begin{cases}1+4 T_{S D O F} & 0 \leq T_{S D O F} \leq 0.15 \mathrm{~s} \\ 1.6 & T_{S D O F} \geq 0.15 \mathrm{~s}\end{cases}
$$

The expression is independent of $\theta-\alpha$, viscous damping in the range $0 \leq \zeta \leq 0.05$, and $T_{S D O F} \geq 0.15 \mathrm{~s}$. Additionally, the averaged IM based on the selected $\varepsilon_{\alpha}$ also leads to an efficient IM when using the peak-oriented hysteretic model instead of the bilinear model (Tsantaki 2014). Subsequently, for $T_{S D O F} \geq 0.15 \mathrm{~s}$ the corresponding averaged IM $S_{a, g m}\left(T_{1}, T_{n, \alpha}\right)$, with the elongated period

$$
T_{n, \alpha}=\varepsilon_{\alpha} T_{S D O F}
$$

is denoted as $S_{a, g m}^{\alpha}\left(T_{S D O F}, 1.6 T_{S D O F}\right)$, or briefly $S_{a, g m}^{\alpha}$.

\section{4 "P-delta" intensity measure}

In this section, the dispersion of the $5 \%$ damped pseudo-acceleration response spectra $S_{a}^{(i)}$, $i=1, \ldots, 44$, of the 44 ground motions of the FEMA P-695-FF is studied when scaled to the "P-delta" IM $S_{a}\left(T_{S D O F}^{P \Delta}\right)$ and to the conventional IM $S_{a}\left(T_{S D O F}\right)$. Figure 12a shows the $5 \%$ damped pseudo-acceleration response spectra $S_{a}^{(i)}\left(T_{S D O F}\right)$ scaled at the period (without P-delta) $T_{S D O F}=3.0 \mathrm{~s}$. Assuming a large stability coefficient of $\theta=0.40, T_{S D O F}^{P \Delta}=$ $T_{S D O F} \sqrt{1 /(1-\theta)}$ becomes $3.87 \mathrm{~s}$. At $T_{S D O F}^{P \Delta}$ the dispersion of the response spectra, $\sigma_{\ln S a}$, scaled to $T_{S D O F}$ is already 0.35 , see Fig. 11a. Thus, even in the elastic range a distinct dispersion of the system response can be observed. To correct this additional variability in the records, Fig. 12b shows the corresponding scaled spectra based on $S_{a}^{(i)}\left(T_{S D O F}^{P \Delta}\right)$. The same reference spectral acceleration equal to $0.1 \mathrm{~g}$ was used for both IMs.

Figure 13 shows RTR dispersion spectra $s^{*}$ based on the "P-delta" IM $S_{a}\left(T_{S D O F}^{P \Delta}\right)$ for negative post-yield stiffness ratios $\theta-\alpha$ of $0.04,010,0.20,0.30,0.40,0.60$ and 0.80 . With increasing $\theta-\alpha$ the system becomes more prone to collapse due to the steep negative post-yielding slope, and less dependent on RTR variability. It is observed that IM $S_{a}\left(T_{S D O F}^{P \Delta}\right)$ reflects appropriately this physical behavior.

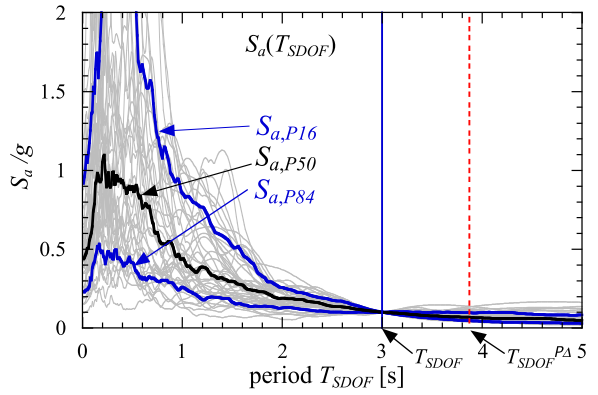

(a)

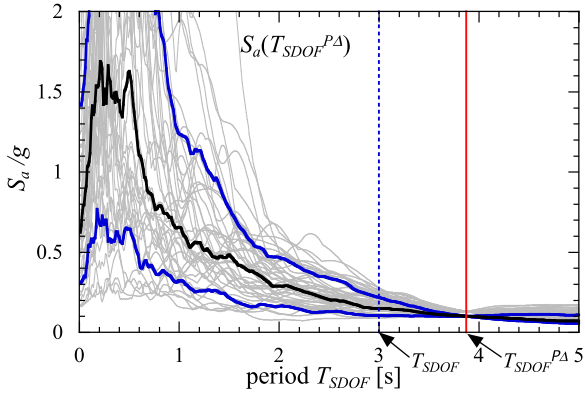

(b)

Fig. 12 Scaled individual record dependent elastic pseudo-acceleration response spectra and their corresponding 50th, 16th and 84th percentiles for a $S_{a}\left(T_{S D O F}\right)$, b $S_{a}\left(T_{S D O F}^{P \Delta}\right)$ 


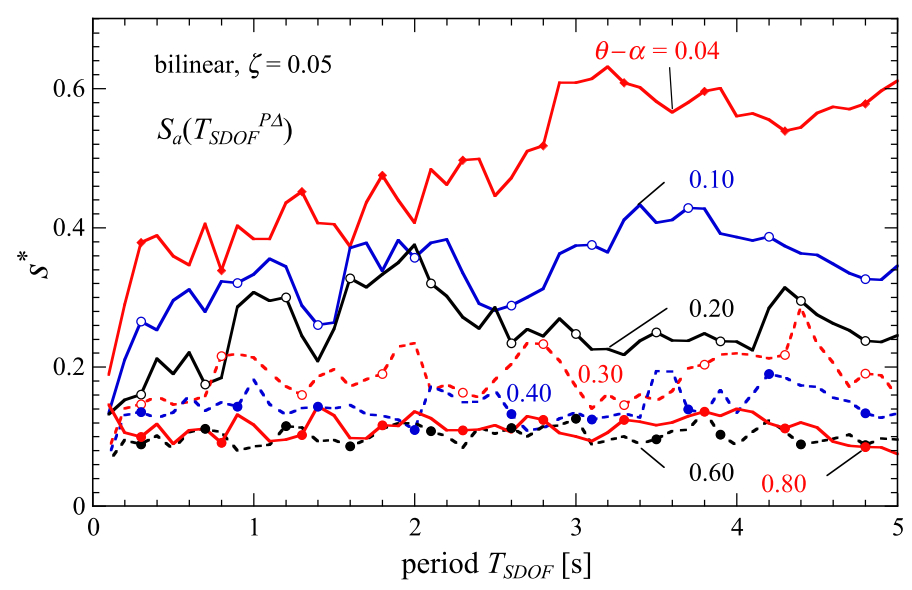

Fig. 13 RTR dispersion spectra $s^{*}$ for different negative post-yield stiffness ratios $\theta-\alpha$ based on IM $S_{a}\left(T_{S D O F}^{P \Delta}\right)$

\section{A comparison of the efficiency of different intensity measures}

In this section, the effect of the RTR variability on the collapse capacity variability of Pdelta vulnerable SDOF systems is compared for the "optimal" averaged IM $S_{a, g m}^{\alpha}$ and two single target pseudo-spectral acceleration IMs: the "P-delta" IM $S_{a}\left(T_{S D O F}^{P \Delta}\right)$ and the benchmark IM $S_{a}\left(T_{S D O F}\right)$. System configurations for 50 values of $T_{S D O F}=0.1,0.2,0.3, \ldots, 5 \mathrm{~s}$., and 13 cases of $\theta-\alpha=0.04,0.06,0.08,0.10,0.15,0.20$, $0.25,0.30,0.35,0.40,0.45,0.60$ and 0.80 are considered, using a damping value $\zeta=0.05$.

Figure 14 presents the dispersion $s^{*}$ spectra for the three studied IMs and nine $\theta-\alpha$ values. For small negative post-yield stiffness ratios; i.e., $\theta-\alpha \leq 0.10$, the two single target IMs yield similar RTR dispersion spectra that are larger than those based on the averaged IM $S_{a, g m}^{\alpha}$. The reduction of RTR variability based on IM $S_{a, g m}^{\alpha}$ becomes more evident when the post-yield stiffness increases up to 0.20 . Also, the efficiency of IM $S_{a}\left(T_{S D O F}^{P \Delta}\right)$ starts to increase noticeably for $\theta-\alpha>0.45$. For $0.30 \leq \theta-\alpha \leq 0.45$ the collapse capacity dispersion based on IM $S_{a}\left(T_{S D O F}^{P \Delta}\right)$ is shifted to smaller RTR values leading to similar spectra based on the IM $S_{a, g m}^{\alpha}$. For $\theta-\alpha=0.60$ and 0.80 , the IM $S_{a}\left(T_{S D O F}^{P \Delta}\right)$ is the most efficient IM.

These trends are verified in Fig. 15, which summarizes the mean dispersions $\mathrm{E}_{T_{S D O F}}\left[s^{*}\right]$ and $\mathrm{E}_{\theta-\alpha}\left[s^{*}\right]$ for the three studied IMs. As observed, $\mathrm{E}_{T_{S D O F}}\left[s^{*}\right]$ and $\mathrm{E}_{\theta-\alpha}\left[s^{*}\right]$ are significantly larger for the benchmark IM $S_{a}\left(T_{S D O F}\right)$. Figures 15a shows the mean dispersion $\mathrm{E}_{T_{S D O F}}\left[s^{*}\right]$ of $s^{*}$, as a function of $\theta-\alpha$, with respect to 50 values of periods $T_{S D O F}$. For $\theta-\alpha \leq 0.10$ the period elongation is mainly due to the potential of large inelastic deformations. Hence, the only IM that reduces the collapse capacity dispersion is $S_{a, g m}^{\alpha}$.

As $\theta-\alpha$ increases, the systems become more prone to failure, and less dependent on the frequency content of the ground motions. Therefore, for very steep negative post-yield stiffness, collapse capacity dispersion is less affected by RTR variability. The expected behavior of increasing efficiency with increasing $\theta-\alpha$ is only observed for outcomes based on the IM $S_{a}\left(T_{S D O F}^{P \Delta}\right)$. 


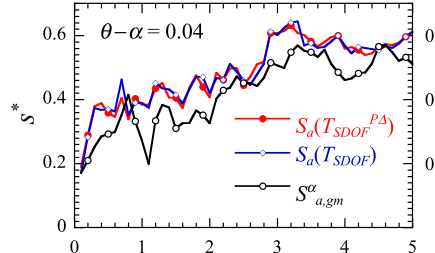

(a)

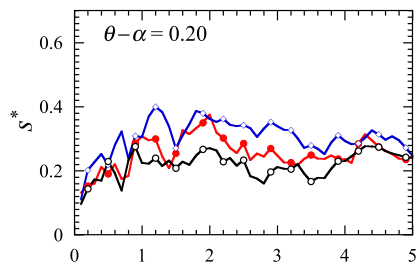

(d)

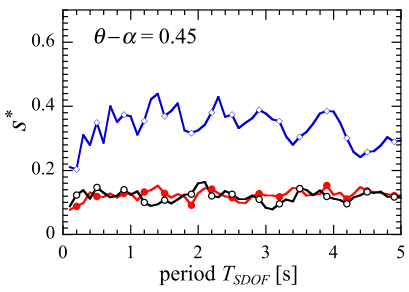

(g)

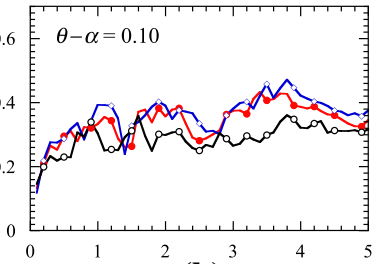

(b)

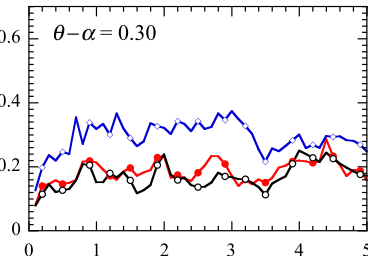

(e)

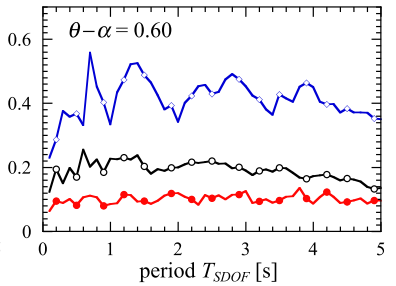

(h)

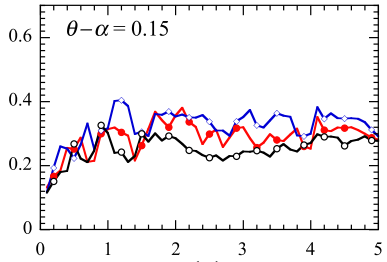

(c)

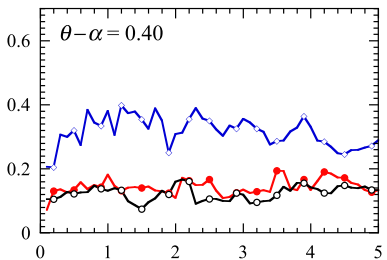

(f)

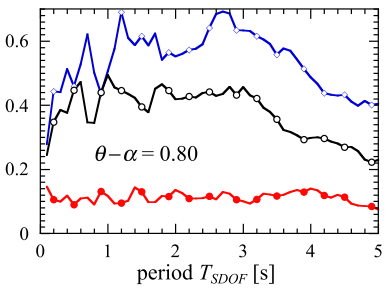

(i)

Fig. 14 RTR dispersion $s^{*}$ spectra. IMs and negative post-yield stiffness ratios $\theta-\alpha$ as specified

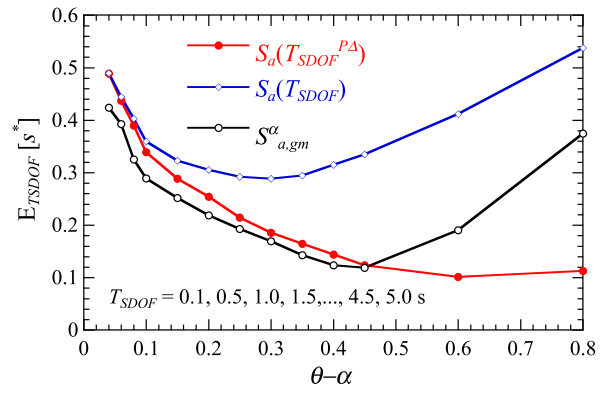

(a)

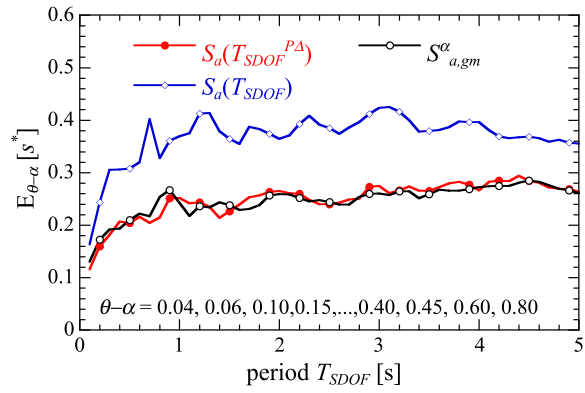

(b)

Fig. 15 Mean dispersion measures a $E_{T_{S D O F}}\left[s^{*}\right]$ and $\mathbf{b} E_{\theta-\alpha}\left[s^{*}\right]$. IMs, negative post-yield stiffness ratios $\theta-\alpha$ and system periods $T_{S D O F}$ as specified

A contradicting trend is observed for IM $S_{a}\left(T_{S D O F}\right)$ if $\theta-\alpha \geq 0.30$, and for the IM $S_{a, g m}^{\alpha}$ if $\theta-\alpha \geq 0.45$. As $\theta-\alpha$ increases, the difference between the periods $T_{S D O F}$ and $T_{S D O F}^{P \Delta}$ becomes so large that the dispersion starts to increase. At $\theta=0.41$ period $T_{S D O F}^{P \Delta}$ is about 1.3 times $T_{S D O F}$, and thus, it is in the center of averaging period interval $\Delta T$. Consequently, at $\theta-\alpha \approx 0.45$ the efficiency of $\operatorname{IM} S_{a, g m}^{\alpha}$ and $\operatorname{IM} S_{a}\left(T_{S D O F}^{P \Delta}\right)$ is about the 
same because "higher mode effects" and effect of period elongation cancel out. Further increase of $\theta-\alpha(>0.45)$ impairs the efficiency of IM $S_{a, g m}^{\alpha}$.

It can be concluded that IM $S_{a, g m}^{\alpha}$ is the most efficient IM for $\theta-\alpha \leq 0.45$. However, for larger $\theta-\alpha$ values, IM $S_{a}\left(T_{S D O F}^{P \Delta}\right)$ leads to the smallest collapse capacity dispersion. Figure $15 \mathrm{~b}$ shows the mean with respect to 13 values of $\theta-\alpha$ as a function of $T_{\text {SDOF. }}$. A fluctuation of the mean collapse capacity variability $\mathrm{E}_{\theta-\alpha}\left[s^{*}\right]$ around 0.23 is observed for relative IMs $S_{a, g m}^{\alpha}$ and $S_{a}\left(T_{S D O F}^{P \Delta}\right)$, as opposed to 0.37 for the IM $S_{a}\left(T_{S D O F}\right)$.

Note that an averaged IM, where the lower bound period corresponds to P-delta affected period; i.e. $T_{1}=T_{S D O F}^{P \Delta}$, is not more efficient than IM $S_{a, g m}^{\alpha}\left(T_{S D O F}, 1.6 T_{S D O F}\right)$ proposed in Sect. 3.3. Additionally, for such IM the ratio between the upper bound and lower bound period is not constant, but depends strongly on the negative post-yield stiffness ratio and actual system period. Consequently, the definition of a simple "global" IM with $T_{1}=$ $T_{S D O F}^{P \Delta}$ valid for a wide range of system parameters is not possible (Tsantaki 2014).

\section{Sufficiency and scaling robustness of the utilized intensity measures}

The sufficiency and scaling robustness properties of the utilized IMs with respect to the natural logarithm of the 44 collapse capacities of the FEMA P-695-FF record set are examined for 500 structural configurations with system periods $T_{S D O F}=0.1,0.2,0.3, \ldots$, 4.8, 4.9, $5.0 \mathrm{~s}$, and post-yield stiffness ratios $\theta-\alpha=0.04,0.06,0.08,0.10,0.20,0.30$, $0.40,0.50,0.60,0.80$. The sufficiency property is studied for two ground motion characteristics: the earthquake magnitude $(M)$, and the natural logarithm of the source-to-site distance (Bozorgnia and Campbell 2004; Bommer and Akkar 2012). The selected measures of source-to-site distance are the epicentral distance $R_{e p i}$ as a point-source distance metric, and the Joyner-Boore distance $R_{J B}$ as an extended-source distance metric. The present evaluation on the linear or logarithmic dependencies of the natural logarithm of the collapse capacities is consistent with the studies of Cornell et al. (1979), Abrahamson and Silva (1997), Bianchini et al. (2009), and Bojórquez and Iervolino (2011) on the considered ground motion characteristics, such as the magnitude, the natural logarithm of the sourceto-site distance, and the natural logarithm of the scaling factors.

Initially, scatter plots have been used to explore by visual inspection the possible linear or non-linear relationship between paired sets consisting of the natural logarithm of the collapse capacities and the considered parameter. For instance, Fig. 16 shows the scatter plots of the natural logarithm of the 44 collapse capacities of the FEMA P-695-FF record set for two system configurations, $T_{S D O F}=1.0 \mathrm{~s}, \theta-\alpha=0.10$, and $T_{S D O F}=3.0 \mathrm{~s}$, $\theta-\alpha=0.20$, for all the utilized IMs as a function of the magnitude $M$. Figure 17 shows similar scatter plots as a function of the scaling factors $S F$. Note that these figures show the non-logarithmic values plotted in logarithmic scaled axes (for logarithmic quantities). The scatter plots of all the considered structural configurations have not revealed any apparent non-linear relationship. Therefore, only a linear relationship is further examined. Firstly, linear regression analyses were conducted, and the linear regression slope coefficients $\beta$ estimated, where a $\beta$ value close to 0 indicates negligible correlation of the two paired sets. For each parameter set and IM of Figs. 16 and 17, the corresponding linear regression fit and estimate of the linear regression coefficient $\beta$ are specified.

In a further step the potential correlation between the evaluated parameters for all the considered structural configurations is quantified in terms of the linear correlation 


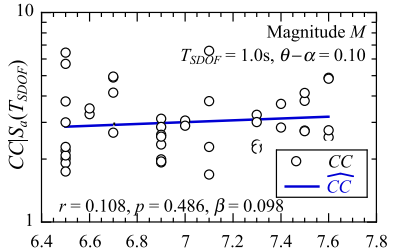

(a)

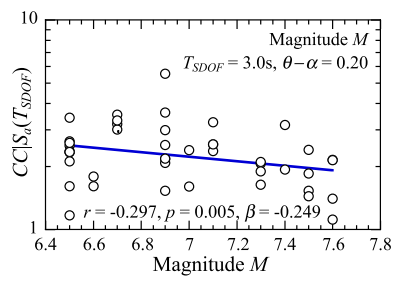

(d)

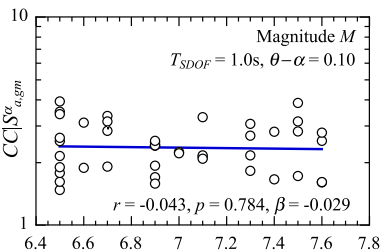

(b)

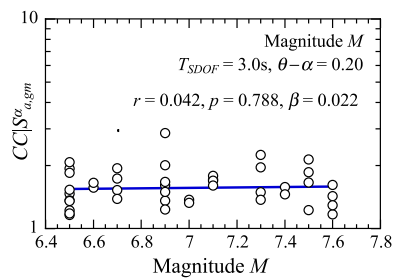

(e)

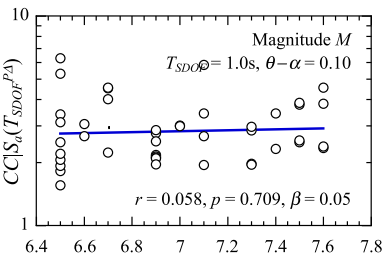

(c)

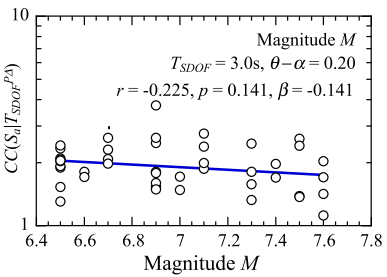

(f)

Fig. 16 Scatter plots and linear regression fits about the sufficiency property with respect to the earthquake magnitude $M$ for IM a, $\mathbf{d} S_{a}\left(T_{S D O F}\right)$, b, e $S_{a, g m}^{a} \mathbf{c}, \mathbf{f} S_{a}\left(T_{S D O F}^{P \Delta}\right)$ and the 44 collapse capacities of the FEMA P$695-\mathrm{FF}$ record set for two system configurations (a-c) $T_{S D O F}=1.0 \mathrm{~s}, \theta-\alpha=0.10$ and (d-f) $T_{\text {SDOF }}=3.0 \mathrm{~s}, \theta-\alpha=0.20$

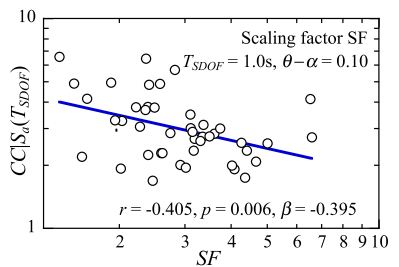

(a)

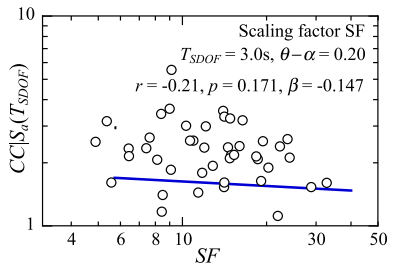

(d)

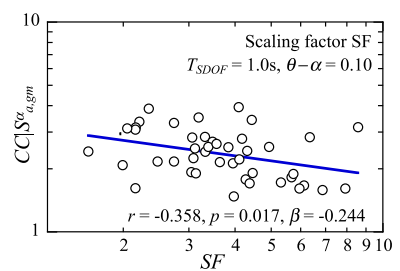

(b)

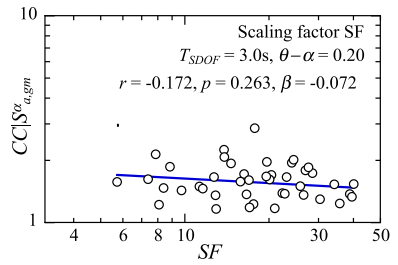

(e)

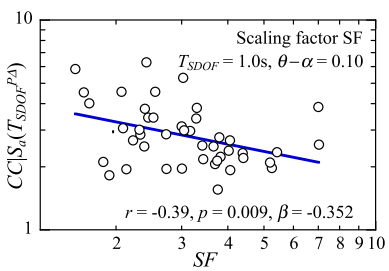

(c)

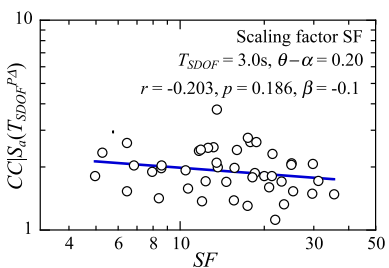

(f)

Fig. 17 Scatter plots and linear regression fits about the scaling robustness property for IM a, $\mathbf{d} S_{a}\left(T_{S D O F}\right)$, $\mathbf{b}, \mathbf{e} S_{a, g m}^{a}, \mathbf{c}, \mathbf{f} S_{a}\left(T_{S D O F}^{P \Delta}\right)$ with respect to the scaling factors and the 44 collapse capacities of the FEMA P$695-\mathrm{FF}$ record set for two system configurations a-c $T_{S D O F}=1.0 \mathrm{~s}, \theta-\alpha=0.10$ and $\mathbf{d}-\mathbf{f}$ $T_{S D O F}=3.0 \mathrm{~s}, \theta-\alpha=0.20$

coefficient $r$ and the $p$ value function. The linear correlation coefficient $r$ measures the strength and direction of a linear regression between two variables, and it is computed from the covariance of the two variables divided by the product of their standard deviations. Correlation coefficient $r$ varies from -1 to 1 (Fenton and Neil 2012), where values close to 0 indicate a negligible correlation, values smaller than 0.5 a weak correlation, and values 
larger than 0.8 a strong correlation (Roberts and Roberts 2015). The $p$ value determines the "statistical significance" of the correlation coefficients $r$, representing the probability of obtaining a correlation as large as the observed linear correlation coefficient by random chance when the true correlation is zero (MATLAB and Statistics Toolbox Release 2015a, 2015). Since a $p$ value is a probability quantity, it ranges from 0 to 1 (GraphPad Statistics Guide 2015). A threshold $p$ value equal to 0.05 was predefined before conducting the statistical test. A $p$ value smaller than this threshold indicates the "statistical significance" of the observed values and the rejection of the null hypothesis of non-correlation. This means that the computed $r$-values would rarely occur (i.e., with a probability equal to 0.05 ) due to random sampling if the two paired sets were correlated from identical populations. For the considered system configurations, IMs, and paired sets in Figs. 16 and 17 also the corresponding correlation coefficient $r$ and the $p$ value are specified.

Figures 18, 19, 20 and 21 show the correlation coefficients $r$ and $p$ values as a function of $T_{S D O F}$ for the negative post-yield stiffness ratios $\theta-\alpha=0.04,0.10,0.20,0.30,0.40$ and 0.80 . Figures 18, 19 and 20 reveal that the correlation coefficients $r$ with respect to the sufficiency property ranges in general from -0.25 to 0.25 for all considered ground motion parameters, IMs, and $\theta-\alpha$ values. Exceptions are larger $r$ values for the extremely large negative post-yield stiffness ratio $\theta-\alpha=0.80$, and a few structural parameter configurations with steep post-yield negative slopes, such as $\theta-\alpha=0.30$ and 0.40 , in particular for the benchmark IM $S_{a}\left(T_{S D O F}\right)$. In the period range $0<T_{S D O F}<2 \mathrm{~s}$, the $r$-value fluctuates around 0 , in contrast to longer system periods where larger $r$-values are observed. Correspondingly, the $p$ values are in general above the threshold 0.05 for the majority of configurations, except for $\theta-\alpha=0.80$ and a few additional $\theta-\alpha$ cases in system period domains with values larger than $2.0 \mathrm{~s}$. As observed, the system period regions with $r$ values larger than 0.25 correspond, in general, to the system period domains with a $p$ value smaller than 0.05 . These system period domains depend on the ground motion characteristics and the underlying IM. For example, the period domain is $T_{S D O F}>3.0 \mathrm{~s}$ for magnitude $M$ and IM $S_{a}\left(T_{S D O F}\right), T_{S D O F}>4.0 \mathrm{~s}$ for $M$ and IM $S_{a, g m}^{\alpha}, T_{S D O F}>2.5 \mathrm{~s}$ for

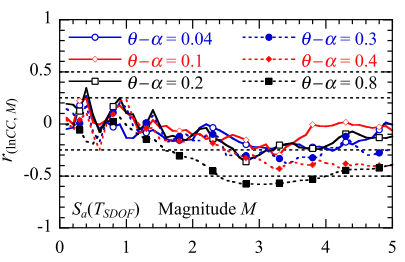

(a)

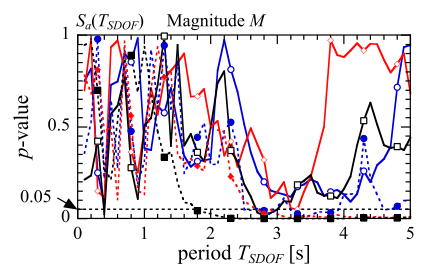

(d)

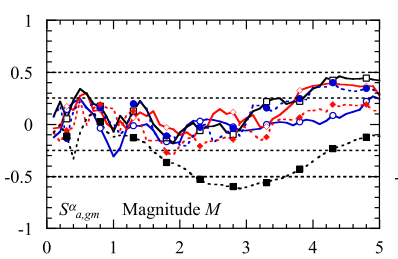

(b)

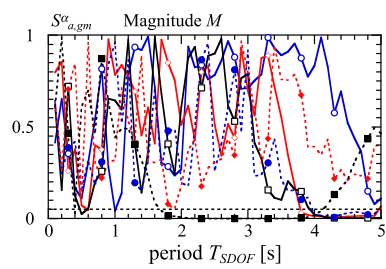

(e)

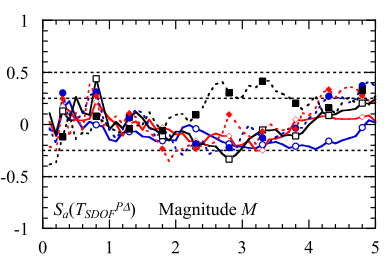

(c)

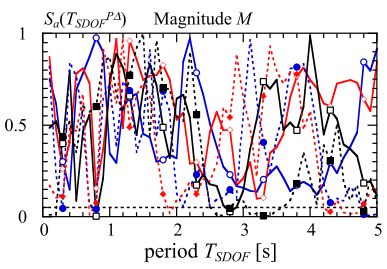

(f)

Fig. 18 a-c Correlation coefficient and $\mathbf{d}-\mathbf{f} p$ value plots as a function of system period $T_{S D O F}$ about the sufficiency property of a, d $S_{a}\left(T_{S D O F}\right), \mathbf{b}, \mathbf{e} S_{a, g m}^{a}, \mathbf{c}, \mathbf{f} S_{a}\left(T_{S D O F}^{P \Delta}\right)$ IM with respect to the earthquake magnitude (M). Negative post-yield stiffness ratios $\theta-\alpha$ as specified 


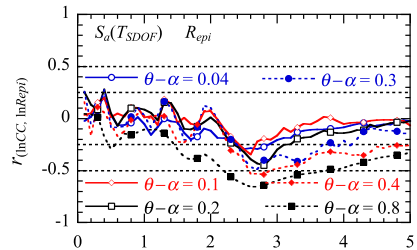

(a)

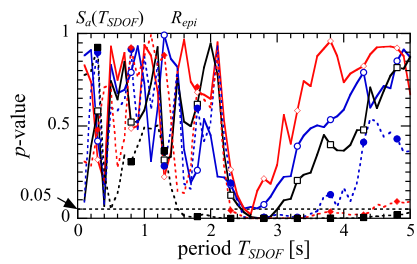

(d)

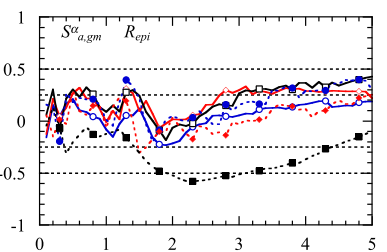

(b)

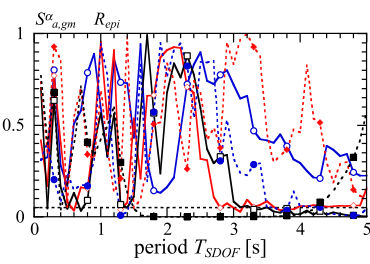

(e)

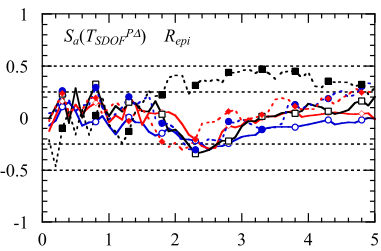

(c)

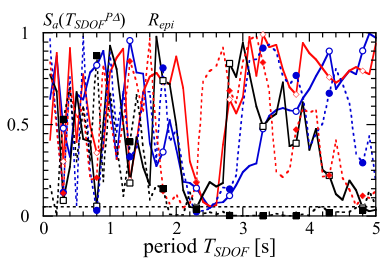

(f)

Fig. 19 a-c Correlation coefficient and $\mathbf{d}-\mathbf{f} p$ value plots as a function of system period $T_{S D O F}$ about the sufficiency property of a, d $S_{a}\left(T_{S D O F}\right)$, b, e $S_{a, g m}^{a}, \mathbf{c}, \mathbf{f} S_{a}\left(T_{S D O F}^{P \Delta}\right)$ IM with respect to the epicentral distance $\left(R_{e p i}\right)$. Negative post-yield stiffness ratios $\theta-\alpha$ as specified

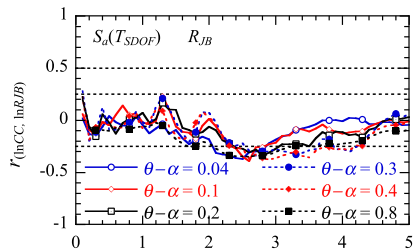

(a)

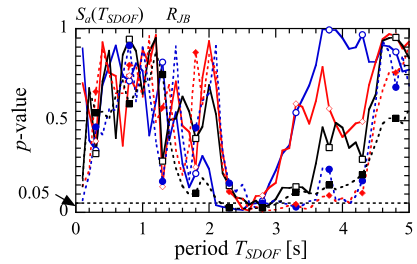

(d)

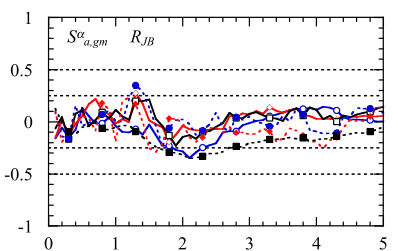

(b)

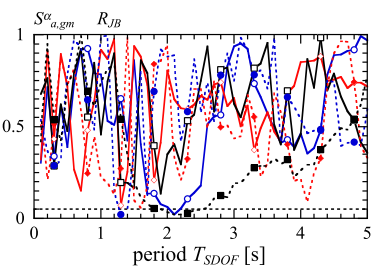

(e)

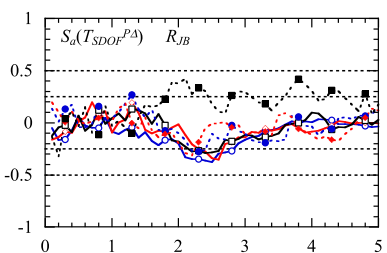

(c)

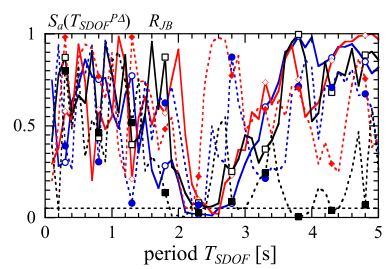

(f)

Fig. 20 a-c Correlation coefficient and $\mathbf{d}-\mathbf{f} p$ value plots as a function of system period $T_{S D O F}$ about the sufficiency property of a, d $S_{a}\left(T_{S D O F}\right), \mathbf{b}, \mathbf{e} S_{a, g m}^{a}$, c, f $S_{a}\left(T_{S D O F}^{P \Delta}\right)$ IM with respect to the Joyner-Boore distance $\left(R_{J B}\right)$. Negative post-yield stiffness ratios $\theta-\alpha$ as specified

point-source distance metric $R_{e p i}$ and $S_{a}\left(T_{S D O F}\right), T_{S D O F}>4.0 \mathrm{~s}$ for $R_{e p i}$ and IM $S_{a, g m}^{\alpha}$, $2.0 \mathrm{~s}<T_{S D O F}<3.5 \mathrm{~s}$ for the extended-source distance metric $R_{J B}$ and IM $S_{a}\left(T_{S D O F}\right)$, and $2.0 \mathrm{~s}<T_{S D O F}<2.8 \mathrm{~s}$ for $R_{J B}$ and $S_{a}\left(T_{S D O F}^{P \Delta}\right)$. In all other combinations just a few narrow banded and scattered period domains are observed.

The correlation coefficients $r$ with respect to scaling robustness property is expressed by the relation between the natural logarithm of the collapse capacities and the natural logarithm of the scaling factors. In general, this coefficient ranges between -0.50 and 0.0 for 


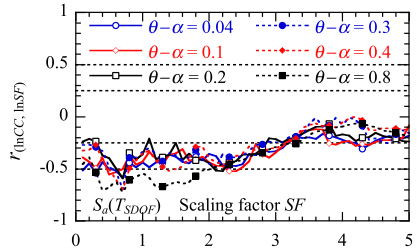

(a)

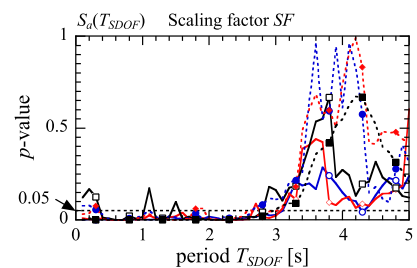

(d)

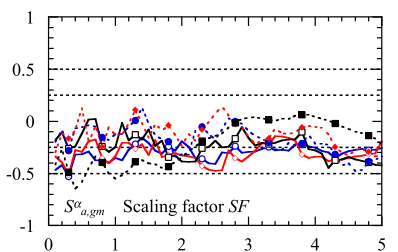

(b)

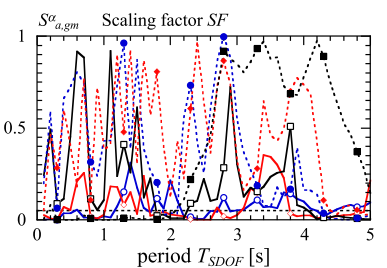

(e)

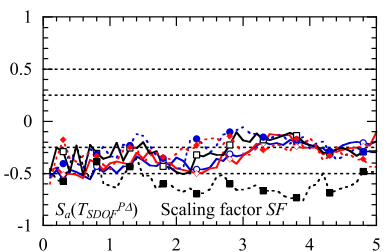

(c)

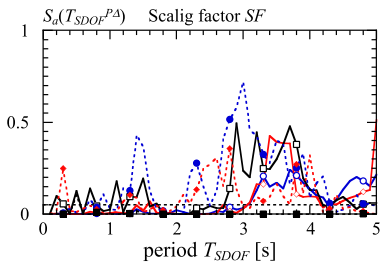

(f)

Fig. 21 a-c Correlation coefficient and $\mathbf{d}-\mathbf{f} p$ value plots as a function of system period $T_{S D O F}$ about the scaling robustness property of a, d $S_{a}\left(T_{S D O F}\right) \mathbf{b}, \mathbf{e} S_{a, g m}^{a} \mathbf{c}, \mathbf{f} S_{a}\left(T_{S D O F}^{P \Delta}\right)$ IM. Post-yield stiffness ratios $\theta-\alpha$ as specified

all considered IMs and system configurations, except for systems with $\theta-\alpha=0.80$ (Fig. 21). For both single-target IMs, $r$ is around 0.35 if $T_{S D O F}<3 s$. In contrast, in this period range for the averaged IM $S_{a, g m}^{\alpha}$ the $r$-value is only about 0.20 . For system periods $T_{S D O F}>3 s$ the collapse capacities based on the benchmark IM exhibit smaller correlation coefficients $r$ varying from 0 to -0.25 , while for the "P-delta" and the averaged IM $r$ is in general about 0.25 , except for a few cases of $\theta-\alpha$ values. Also, $p$ values less than 0.05 are observed for all IMs, however, in case of the single target IMs the corresponding period regions are more extended and include larger numbers of the considered $\theta-\alpha$ values.

The $p$ values in Figs. 18, 19, 20 and 21 below the selected threshold of 0.05 indicate that the computed $r$ values are "statistical significant". For such cases, the selected IM is conditionally linearly dependent with respect to the natural logarithm of collapse capacities on the considered parameters, such as ground motion characteristics and scaling factors. Therefore, these period domains do not strictly meet the properties of sufficiency and scaling robustness. However, the period intervals in which $p$ values are below the predefined threshold of 0.05 are very limited, except for the scaling robustness property (Fig. 21). More important, in all cases the correlation coefficient is smaller than $r<0.5$, indicating a weak correlation between the selected IM and the ground motion characteristics or scaling factors.

For instance, the natural logarithm of scaling factors is weakly correlated $(r<0.5)$ with the natural logarithm of the 44 collapse capacities of the FEMA P-695-FF record set, and for some scenarios it is statistically significant $(p$ value $<0.05)$. This correlation is negative (Figs. 17 and 21), revealing a decrease of the natural logarithm of collapse capacities as the natural logarithm of scaling factors increases. This finding is consistent with results of Bianchini et al. (2009). In all other cases with $p$ values larger than the selected threshold, the correlation is considered as "statistical insignificant". 


\section{Conclusions}

Two alternative elastic pseudo-spectral acceleration based IMs were compared with the widely accepted IM, $S_{a}\left(T_{S D O F}\right)$; i.e., the $5 \%$ damped pseudo-spectral acceleration at the system period $T_{S D O F}$ of the structure without P-delta. The efficiency of the two alternative IMs to reduce collapse capacity dispersion caused by record-to-record (RTR) variability was presented. Initially, three different sets of averaged IMs were studied with different lower and upper bounds of the period interval to obtain the "optimal" averaged IM that leads to the smallest collapse capacity dispersion due to RTR variability. From the results of this parametric study in a wide range of structural configurations, the following conclusions can be drawn for the considered type of P-delta vulnerable and non-deteriorating simple systems:

- The lower bound of the period interval corresponds to the SDOF period of vibration because no higher modes effects do exist. This result cannot be transferred to multidegree-of-freedom (MDOF) structures.

- The "optimal" upper bound of the period interval for the averaging IM is on average about 1.6 times the system period. The period elongation is either a result of large inelastic deformations in the case of small negative post-yield stiffness slopes, or the results of the presence of gravity loads in systems with steeper negative slopes.

- An "optimal" averaged IM $S_{a, g m}^{\alpha}\left(T_{S D O F}, 1.6 T_{S D O F}\right)$, or briefly $S_{a, g m}^{\alpha}$ is introduced for system periods larger than $0.15 \mathrm{~s}$, which is composed of the geometric mean of the $5 \%$ damped spectral acceleration in the range between the system period and 1.6 times this period. Note that the upper limit of this period range is similar to values proposed in several guidelines, without being clearly verified by or/and related to previous research studies.

Subsequently, an alternative single target IM is introduced, $S_{a}\left(T_{S D O F}^{P \Delta}\right)$, which corresponds to the $5 \%$ damped pseudo-spectral acceleration at the system period considering gravity load effects. This "P-delta" IM leads to smaller collapse capacity dispersion as the negative post-yield stiffness ratio becomes larger than 0.45 .

The most efficient IM, leading to the smallest RTR dispersion, for systems with negative post-yield stiffness ratios $\theta-\alpha \leq 0.45$ is $S_{a, g m}^{\alpha}$; for systems with $\theta-\alpha>0.45$ the most efficient IM is $S_{a}\left(T_{S D O F}^{P \Delta}\right)$.

For IMs $S_{a, g m}^{\alpha}$ and $S_{a}\left(T_{S D O F}^{P \Delta}\right)$, the mean RTR variability is on average 0.23 , with respect to the considered post-yield stiffness ratios. In contrast, for $S_{a}\left(T_{S D O F}\right)$, this mean is 0.37 ; i.e., about $60 \%$ larger.

The sufficiency and scaling robustness property of the utilized IMs with respect to the natural logarithm of the 44 collapse capacities of the FEMA P-695-FF record set were examined for the considered structural configurations. The sufficiency property was studied for two ground motion characteristics; i.e., the earthquake magnitude and the natural logarithm of the source-to-site distance. Two measures of source-to-site distance were selected: the epicentral distance as a point-source distance metric, and the Joyner-Boore distance as an extended-source distance metric. The scatter plots revealed that there is no apparent non-linear relationship between the natural logarithm of the collapse capacities and the corresponding ground motion characteristics. Therefore, only the potential of linear relationship was further examined, quantifying the linear correlation coefficients $r$ and their corresponding $p$ values. The outcomes can be summarized as follows. 
- For the selected structural configurations the period domains and negative post-yield stiffness ratios $\theta-\alpha$ with $r>0.25$ and $p$ value $<0.05$ were identified for all the considered paired sets and IMs. Note that $p$ values below the selected threshold of 0.05 indicate that the computed $r$ values are "statistically significant". For such cases, the selected IM is conditionally linearly dependent on the considered ground motion parameters or scaling factors (with respect to the natural logarithm of collapse capacities), and may not meet the properties of sufficiency and scaling robustness. These domains are more extended for the conventional IM $S_{a}\left(T_{S D O F}\right)$.

- The natural logarithm of scaling factors exhibits a weak correlation with the natural logarithm of the collapse capacities. This correlation is negative, revealing a decrease of the natural logarithm of collapse capacities as the natural logarithm of scaling factors increases.

The presented results are only valid for single-degree-of-freedom systems and should not be extrapolated to multi-degree-of-freedom structures.

Acknowledgments S. Tsantaki conducted the research for this paper as student of the Doctoral School (FWF DK-plus) "Computational Interdisciplinary Modelling", which is based at the University of Innsbruck and substantially funded by the Austrian Science Fund (FWF). Open access funding provided by University of Innsbruck and Medical University of Innsbruck.

Open Access This article is distributed under the terms of the Creative Commons Attribution 4.0 International License (http://creativecommons.org/licenses/by/4.0/), which permits unrestricted use, distribution, and reproduction in any medium, provided you give appropriate credit to the original author(s) and the source, provide a link to the Creative Commons license, and indicate if changes were made.

\section{References}

Abrahamson NA, Silva WJ (1997) Empirical response spectral attenuation relations for shallow crustal earthquakes. Seismol Res Lett 68(1):94-127

Adam C, Jäger C (2012a) Seismic collapse capacity of basic inelastic structures vulnerable to the P-delta effect. Earthq Eng Struct Dyn 41:775-793

Adam C, Jäger C (2012b) Dynamic instabilities of simple inelastic structures subjected to earthquake excitation. In: Irschik H, Krommer M, Belyaev A (eds) Advanced dynamics and model-based control of structures and machines. Springer, Wien, pp 11-18

ASCE/SEI 41-13 (2014) Seismic evaluation and retrofit of existing buildings. American Society of Civil Engineers, Reston, Virginia, USA

Baker JW (2007) Measuring bias in structural response caused by ground motion scaling. In: Proceedings of the 8th Pacific conference on earthquake engineering (8PCEE 07), Nangyang Technological University, Singapore, 5-7 Dec 2007

Baker JW, Cornell CA (2005) A vector-valued ground motion intensity measure consisting of spectral acceleration and epsilon. Earthq Eng Struct Dyn 34(10):1193-1217

Baker JW, Cornell CA (2006) Spectral shape, epsilon and record selection. Earthq Eng Struct Dyn 35:1077-1095

Baker JW, Jayaram N (2008) Correlation of spectral acceleration values from NGA ground motion models. Earthq Spectra 24(1):299-317

Bernal D (1987) Amplification factors for inelastic dynamic P- $\Delta$ effects in earthquake analysis. Earthq Eng Struct Dyn 15:635-651

Bianchini M, Diotallevi P, Baker JW (2009) Prediction of inelastic structural response using an average of spectral accelerations. In: Proceedings of the 10th international conference on structural safety and reliability (ICOSSAR 09), Osaka, Japan, 13-19 Sept 2009

Bojórquez E, Iervolino I (2011) Spectral shape proxies and nonlinear structural response. Soil Dyn Earthq Eng 31(7):996-1008

Bommer JJ, Akkar S (2012) Consistent source-to-site distance metrics in ground-motion prediction equations and seismic source models for PSHA. Earthq Spectra 28(1):1-15 
Bozorgnia Y, Campbell KW (2004) Engineering characterization of ground motion. In: Bozorgnia Y, Bertero VV (eds) Earthquake engineering: from engineering seismology to performance-based engineering. CRC Press, Book Chapter 5

Cantagallo C, Camata G, Spacone E, Corotis R (2012) Seismic demand uncertainty provided by ground motion intensity measures. In: Proceedings of the 15th world conference on earthquake engineering (15 WCEE), Digital paper, paper no. 0680, Lisbon, Portugal, 24-28 Sept 2012

Clinton JF, Bradford SC, Heaton TH, Favela J (2006) The observed wander of the natural frequencies in a structure. Bull Seismol Soc Am 96(1):237-257

Cordova PP, Deierlein GG, Mehanny SSF, Cornell CA (2001) Development of two-parameter seismic intensity measure and probabilistic assessment procedure. In: Proceedings of the second US-Japan workshop on performance-based earthquake engineering methodology for reinforced concrete buildings structures; 2001, Sapporo, Japan, pp 187-206

Cornell CA, Banon H, Shakal AF (1979) Seismic motion and response prediction alternatives. Earthq Eng Struct Dyn 7(4):295-315

Eads L, Miranda E, Lignos DG (2015) Average spectral acceleration as an intensity measure for collapse risk assessment. Earthq Eng Struct Dyn 44(12):2057-2073

Eurocode 8 (2004) Design provisions of structures for earthquake resistance. Part 1: General rules, seismic actions and rules for buildings. European Committee for Standardization

FEMA P-695 (2009) Quantification of building seismic performance factors. Federal Emergency Management Agency

FEMA P-58-1 (2012) Seismic performance assessment of buildings. Volume 1 - Methodology. Federal Emergency Management Agency

Fenton NE, Neil M (2012) Risk Assessment and Decision Analysis with Bayesian Networks. CRC Press, ISBN: 9781439809105, ISBN 10: 1439809100, 2012

GraphPad Statistics Guide (2015) Graphpad Software Inc. http://www.graphpad.com/. Accessed 20 March 2015

Haselton CB (2009) Evaluation of ground motion selection and modification methods: predicting median interstory drift response of buildings. PEER Report 2009/01, Pacific Earthquake Engineering Research Center, College of Engineering, University of California, Berkeley

Haselton CB, Baker JW (2006) Ground motion intensity measures for collapse capacity prediction: choice of optimal spectral period and effect of spectral shape. In: Proceedings of the 8th National Conference on Earthquake Engineering, San Francisco, California, 18-22 Apr 2006

Husid R (1967) Gravity effects on the earthquake response of yielding structures. Dissertation, California Institute of Technology, Pasadena, California

Ibarra LF, Krawinkler H (2005) Global collapse of frame structures under seismic excitations. PEER Report 2005/06, University of California at Berkeley, California, Pacific Earthquake Engineering Research Center

Ibarra L, Krawinkler H (2011) Variance of collapse capacity of SDOF systems under earthquake excitations. Earthq Eng Struct Dyn 40:1299-1314

Jäger C, Adam C (2013) Influence coefficients for collapse capacity spectra. J Earthq Eng 17:859-878

Jalayer F, Beck JL, Zareian F (2012) Information-based relative sufficiency of some ground motion intensity measures. In: Proceedings of the 15th world conference on earthquake engineering (15 WCEE), Digital paper, paper no. 5176, Lisbon, Portugal, 24-28 Sept 2012

Kadas K, Yakut A, Kazaz I (2011) Spectral ground motion intensity based on capacity and period elongation. J Struct Eng 137(3):401-409

Katsanos EI, Sextos AG, Elnashai AS (2012) Period elongation of nonlinear systems modeled with degrading hysteretic rules. In: Proceedings of the 15th world conference on earthquake engineering (15 WCEE), Digital paper paper no. 1887, Lisbon, Portugal, 24-28 Sept 2012

Kazantzi A, Vamvatsikos D (2015) Intensity measure selection for vulnerability studies of building classes. Earthq Eng Struct Dyn 44:2677-2694

Krawinkler H, Zareian F, Lignos DG, Ibarra LF (2009) Prediction of collapse of structures under earthquake excitations. In: Papadrakakis M, Lagaros, ND, Fragiadakis M (eds) Proceedings of the 2nd international conference on computational methods in structural dynamics and earthquake engineering (COMPDYN 2009) June 22-24, 2009, Rhodes, Greece. CD-ROM paper, paper no. CD449

Limpert E, Stahel WA, Abbt M (2001) Log-normal distributions across the sciences: keys and clues. Bioscience 51:341-352

Lin T, Haselton CB, Baker JW (2013a) Conditional spectrum-based ground motion selection. Part I: hazard consistency for risk-based assessments. Earthq Eng Struct Dyn 42(11):1847-1865 
Lin T, Haselton CB, Baker JW (2013b) Conditional spectrum-based ground motion selection. Part II: intensity-based assessments and evaluation of alternative target spectra. Earthq Eng Struct Dyn 42(11): $1867-1884$

Luco N, Cornell CA (2007) Structure-specific scalar intensity measure for near-source and ordinary earthquake motions. Earthq Spectra 23(2):357-391

MacRae GA (1994) P- $\Delta$ effects on single-degree-of-freedom structures in earthquakes. Earthq Spectra 10:539-568

MATLAB and Statistics Toolbox Release 2015a (2015) The MathWorks Inc. Natick, MA, USA

Mucciarelli M, Masi A, Gallipoli MA, Harabaglia P, Vona M, Ponzo F, Dolce M (2004) Analysis of RC building dynamic response and soil-building resonance based on data recorded during a damaging earthquake (Molise, Italy, 2002). Bull Seismol Soc Am 94:1943-1953

Mucciarelli M, Vona M, Ditommaso R, Gallipoli MR (2012) Experimental measurement of fundamental periods of damaged R.C. buildings. In: Proceedings of the 15th world conference on earthquake engineering (15 WCEE), Digital paper, paper no. 2223, Lisbon, Portugal, 24-28 September 2012

NZSEE (2006) Assessment and improvement of the structural performance of buildings in earthquake. Recommendations of a NZSEE study Group on Earthquake Risk Buildings. New Zealand Society for Earthquake Engineering, New Zealand

O'Donnell AP, Kurama YC, Kalkan E, Taflanidis AA, Beltsar OA (2013) Ground motion scaling methods for linear-elastic structures: an integrated experimental and analytical investigation. Earthq Eng Struct Dyn 42:1281-1300

Pinho R, Elnashai AS (2000) Dynamic collapse testing of a full-scale four storey RC frame. ISET J Earthq Technol 37(4):143-163

Roberts F, Roberts D (2015) Finding your way around statistics 2-Correlation coefficient. Fulton, New York USA, MathBits.com. Accessed 20 March 2015

Shome N, Cornell A (1999) Probabilistic seismic demand analysis of nonlinear structures. Report no. RMS35, Research Management System (RMS) Program, Department of Civil Engineering, Stanford University, Stanford, CA

Tsantaki S (2014) A contribution to the assessment of the seismic collapse capacity of basic structures vulnerable to the destabilizing effect of gravity loads, Dissertation, University of Innsbruck, Innsbruck, Austria

Tsantaki S, Adam C (2013) Collapse capacity spectra based on an improved intensity measure. In: Papadrakakis M, Papadopoulos V, Plevris V (eds) Proceedings of the 4th ECCOMAS thematic conference on computational methods in structural dynamics and earthquake engineering (COMPDYN 2013), CDROM paper, paper no. 1382, Kos Island, Greece, 12-14 June 2013

Tsantaki S, Wurzer L, Jäger C, Adam C, Oberguggenberger M (2015) Refined analytical collapse capacity spectra. Iran J Sci Technol Trans Civ Eng 39(C2):253-270

Vamvatsikos D, Cornell CA (2002) Incremental dynamic analysis. Earthq Eng Struct Dyn 31:491-514

Vamvatsikos D, Cornell CA (2005) Developing efficient scalar and vector intensity measures for IDA capacity estimation by incorporating elastic spectral shape information. Earthq Eng Struct Dyn 34:1573-1600. doi:10.1002/eqe. 496

Zembaty Z, Kowalski M, Pospisil S (2006) Dynamic identification of a reinforced concrete frame in progressive states of damage. Eng Struct 28:668-681 